$\mathrm{MZ}-\mathrm{TH} / 12-04$

January 2012

\title{
Yukawa couplings and masses of non-chiral states for the Standard Model on D6-branes on $T^{6} / \mathbb{Z}_{6}^{\prime}$
}

\author{
Gabriele Honecker ${ }^{\curvearrowright}$ and Joris Vanhoof $\diamond, 1$ \\ ${ }^{\odot}$ Institut für Physik (WA THEP), Johannes-Gutenberg-Universität, D-55099 Mainz, \\ Germany Gabriele.Honecker@uni-mainz.de \\ - Theoretische Natuurkunde, Vrije Universiteit Brussel and The International Solvay \\ Institutes, Pleinlaan 2, B-1050 Brussels, Belgium Joris.Vanhoof@vub.ac.be \\ $\diamond$ Institute of Theoretical Physics, K.U.Leuven, Celestijnenlaan 200D, B-3001 Leuven, \\ Belgium
}

\begin{abstract}
The perturbative leading order open string three-point couplings for the Standard Model with hidden $U S p(6)$ on fractional D6-branes on $T^{6} / \mathbb{Z}_{6}^{\prime}$ from [1, 2] are computed. Physical Yukawa couplings consisting of holomorphic Wilsonian superpotential terms times a non-holomorphic prefactor involving the corresponding classical open string Kähler metrics are given, and mass terms for all non-chiral matter states are derived. The lepton Yukawa interactions are at leading order flavour diagonal, while the quark sector displays a more intricate pattern of mixings. While $\mathcal{N}=2$ supersymmetric sectors acquire masses via only two D6-brane displacements - which also provide the hierarchies between up- and down-type Yukawas within one quark or lepton generation -, the remaining vector-like states receive masses via perturbative three-point couplings to some Standard Model singlet fields with vers along flat directions.

Couplings to the hidden sector and messengers for supersymmetry breaking are briefly discussed.
\end{abstract}

\footnotetext{
${ }^{1}$ Aspirant FWO.
} 


\section{Contents}

1 Introduction $\quad 3$

2 Geometry of the Standard Model on $T^{6} / \mathbb{Z}_{6}^{\prime}$ revisited 4

2.1 Geometric setup . . . . . . . . . . . . . . . . . . . 5

2.2 Full particle spectrum . . . . . . . . . . . . . . . . . . . 9

3 Yukawa couplings $\quad 14$

3.1 Superpotential terms . . . . . . . . . . . . . . . . . . . . . . . 14

3.2 Yukawa couplings for the Standard Model on $T^{6} / \mathbb{Z}_{6}^{\prime} \ldots \ldots$. . . . . . . . . 15

3.3 Remarks on phenomenological aspects . . . . . . . . . . . . . 18

3.4 Physical Yukawa couplings and Kähler metrics . . . . . . . . . . . . . . . . 23

4 Masses of non-chiral representations 24

4.1 The vector-like fields in the particle spectrum . . . . . . . . . . . . 25

4.2 Vacuum expectation values of adjoint representations of $U(3)_{a}$ and $U(2)_{b}$. 31

4.3 Vacuum expectation values of symmetric representations of $U(2)_{b}$. . . . 34

5 Couplings to the 'hidden' $U S p(6)_{h}$ sector 36

6 Conclusions and Outlook $\quad 37$

A Tables with localisations of massless matter states 40

B Tables with suppression factors of three-point interactions 43

$\begin{array}{ll}\text { References } & 49\end{array}$ 


\section{Introduction}

The Standard Model gauge group and charged chiral spectrum have been obtained in a variety of globally consistent ( $R R$ tadpole cancelling or Bianchi identity fulfilling plus constraints from K-theory) string compactifications over the past years, on the one hand on orbifolds of type IIA orientifolds [3-9] and of the heterotic string [10-13] and Gepner models [14-16], on the other hand on smooth Calabi-Yau backgrounds in the framework of heterotic strings [17-23] and of F-theory [24]. For all classes of perturbative models, the dimensional reduction of the classical low-energy supergravity limit serves to test the strength of gauge couplings and to derive the closed string moduli potential at leading order, see e.g. [25-27] in the context of D6-branes, and selection rules on the existence of Yukawa interactions are set-up based on charge neutralness and on intersections of cohomology groups [28].

On toroidal orbifold backgrounds, conformal field theory (CFT) techniques render it possible to go a step further and compute the perturbatively exact holomorphic gauge kinetic functions of heterotic [29, 30] and type IIA compactifications with intersecting D6branes $[31-33,2,34,35]^{2}$ In the process of matching the CFT results with the standard supergravity expressions, the Kähler metrics for open string matter fields and the bulk Kähler potential are obtained to lowest order, see [36] for the six-torus and [34, 35] for toroidal orbifolds. For the six-torus, the results agree with an alternative derivation by means of scattering amplitudes [44-46], which allow to compute the physical Yukawa couplings in perturbation theory as a product of the non-holomorphic Kähler potential and Kähler metric contributions times a holomorphic worldsheet instanton sum derived in $[47,48]$. Very recently, it was shown in [49] that certain one-loop corrections to the open string Kähler metrics are absent in globally consistent intersecting D6-brane models.

Phenomenological implications beyond the matter content and gauge couplings on intersecting D6-branes such as the flavour structure have mainly been discussed for the six-torus, see e.g. [50-53], which does, however, not admit globally consistent supersymmetric intersecting D6-brane models. In the present work, we take a first step at filling this gap by computing all leading holomorphic worldsheet instanton contributions to the perturbative Yukawa couplings for the supersymmetric Standard Model with 'hidden' USp $(6)_{h}$ on intersecting D6-branes in the $T^{6} / \mathbb{Z}_{6}^{\prime}$ orientifold background of [1, 2]. In addition, we determine all open string Kähler metrics for the particular model at the classical level in extension of the partial results given in $[34,35]$. The combination of the contributions from the

\footnotetext{
${ }^{2}$ It is also possible to compute non-perturbative corrections to the holomorphic gauge kinetic function from D-brane instantons, see e.g. [36-39]. For D-brane instanton corrections to Yukawa interactions and other superpotential contributions see e.g. some early works [40-42], for an extensive list see the review [43].
} 
smallest allowed holomorphic worldsheet instanton areas with the non-holomorphic factor involving the Kähler metrics gives an estimate on the size of physical Yukawa couplings in the low-energy supergravity theory. Along the way, we identify possible mass terms for all vector-like matter states of the model. Besides the well known mechanism of continuous displacements or Wilson lines, this involves the computation of a large number of per-

turbative three-point interactions in analogy to the Yukawa couplings and a discussion of vacuum expectation values (vevs) of scalar Standard Model singlet fields which contribute to these interactions.

\section{Outline}

The paper is organised as follows: In section 2 we briefly review the geometry of the background and the localisation of matter states of the Standard Model with hidden $U S p(6)_{h}$ on D6-branes on $T^{6} / \mathbb{Z}_{6}^{\prime}$ from [1, 2]. In section 3, three-point couplings are discussed and the selection rules and suppression factors of quark and lepton Yukawa couplings of the model are presented. Yukawa hierarchies and flavour structures are briefly discussed. In section 4, we discuss how the abundant vector-like fields in the spectrum, which do not stem from microscopic $\mathcal{N}=2$ supersymmetric sectors of the D6-brane set-up, acquire masses through three-point couplings to some Standard Model singlets with vers along flat directions. Section 5 contains a brief discussion of couplings to the hidden sector and potential messenger fields for supersymmetry breaking. Finally, section 6 contains our conclusions. The complete tables of localisations of matter states at pairs of D6-branes and all leading open string three-point interactions of the model with both holomorphic and non-holomorphic suppression factors are collected in two appendices.

\section{Geometry of the Standard Model on $T^{6} / \mathbb{Z}_{6}^{\prime}$ revisited}

We briefly review the type IIA $/ \Omega \mathcal{R}$ orientifold geometry of the $\mathbf{A B a}$ lattice and orbifold fixed points on $T^{6} / \mathbb{Z}_{6}^{\prime}$. We introduce the D6-brane configuration of $[1,2]$ which provides the Standard Model spectrum plus a hidden $U S p(6)_{h}$ gauge group and vector-like exotic matter states, and we discuss the different localisations of matter states on the compact space in terms of intersection sectors $x\left(\theta^{k} y\right)$ of (orbifold images of) D6-branes $x, y$ and various intersection points per sector. The localisation on specific orbifold image D6branes such as two right-handed quarks at $a c$ and one at $a\left(\theta^{2} c\right)$ intersections are required in section 3 to state selection rules for Yukawa and other open string three-point couplings, and the exact positions of the intersection points are needed to determine the dominant worldsheet contributions and estimate the size of the corresponding three-point couplings. 


\subsection{Geometric setup}

\subsubsection{Compact space}

The six-dimensional compact space of the $T^{6} / \mathbb{Z}_{6}^{\prime}$ orbifold consists of a six-torus with an additional discrete symmetry, see [54, 1, 2] and also [55-57] in the context of D6-branes in type IIA string theory. A factorisable $T^{6}$ is separated into three different two-tori, $\otimes_{i=1}^{3} T_{(i)}^{2}$, where each of these can be represented by a parallelogram with cyclic coordinates, e.g. $\left(x_{1}, x_{2}\right) \sim\left(x_{1}+n R_{1}, x_{2}+m R_{2}\right)$ with $(n, m) \in \mathbb{Z} \times \mathbb{Z}$. The $\mathbb{Z}_{6}^{\prime}$ orbifold is generated by $\theta$ acting as a rotation on the complex coordinates $z_{i} \equiv x_{2 i-1}+i x_{2 i}(i=1,2,3)$,

$$
\theta: z_{i} \longrightarrow e^{2 \pi i v_{i}} z_{i}, \quad \text { with } \quad \vec{v}=\frac{1}{6}(1,2,-3) \quad \text { for } \quad T^{6} / \mathbb{Z}_{6}^{\prime} .
$$

The orientifold action for the type IIA string theory consists of the worldsheet parity $\Omega$ and an anti-holomorphic involution $\mathcal{R}$,

$$
\mathcal{R}: z_{i} \longrightarrow \bar{z}_{i}
$$

and it acts crystallographically on each two-torus lattice. In figure 1, a schematic representation is given of the three different two-tori $\mathbf{A B a}$ of the model in [1,2]. The position of the $\Omega \mathcal{R}$ invariant orientifold six-planes (O6-planes) is displayed in dashed lines. The orientifold symmetry $\Omega \mathcal{R}$ acts as a reflection over the $\Omega \mathcal{R}$ invariant O6-plane. The $\mathbb{Z}_{6}^{\prime}$ symmetry on the six-torus can be separated into a $\mathbb{Z}_{2}$ and a $\mathbb{Z}_{3}$ part. The $\mathbb{Z}_{2}$ action is generated by the shift vector $3 \vec{v}=\frac{1}{2}(1,0,-1)$ and acts as a point reflection over the origin on the first and third torus. It leaves the second torus invariant. The invariant lattice points on the first torus are denoted by $(1,4,5,6)$ in figure 1 and on the third torus by $(1,2,3,4)$. These labels are in agreement with the convention of [54].

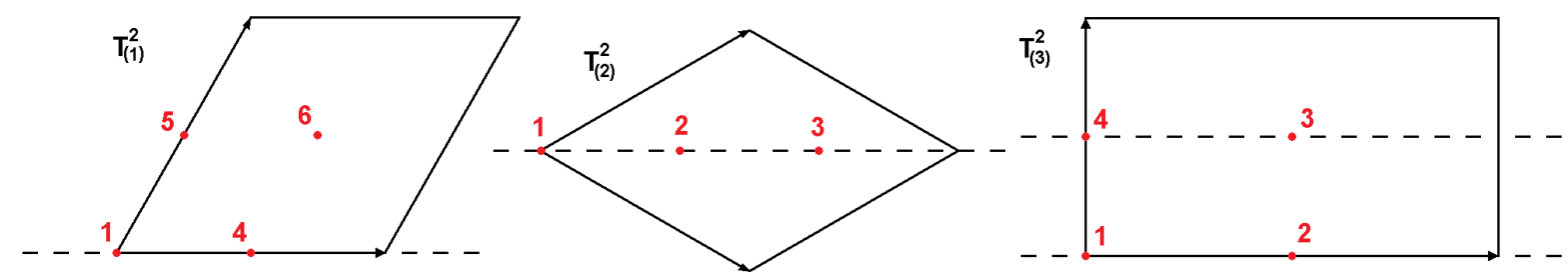

Figure 1: The compact space as a direct product of three two-tori $\mathbf{A B a}$ of $T^{6} / \mathbb{Z}_{6}^{\prime}$. The two $\mathcal{R}$ invariant O6-planes are given by the dashed horizontal lines. On the first and third torus, the marked points are invariant under the $\mathbb{Z}_{2}$ action, while those on the second torus are invariant under a $\mathbb{Z}_{3}$ rotation (with identical copies on the first torus, which are not displayed).

The remaining $\mathbb{Z}_{3}$ action is generated by the shift vector $2 \vec{v}=\frac{1}{3}(1,-1,0)$ and leaves the third torus invariant. On the first torus the images of the $\mathbb{Z}_{2}$ invariant points under the 
$\mathbb{Z}_{6}^{\prime}$ orbifold action are given by

$$
T_{(1)}^{2}: \quad \theta(1)=1, \quad \theta(4)=5, \quad \theta(5)=6, \quad \theta(6)=4 .
$$

The points $(1,2,3)$ on the second torus are invariant under the $\mathbb{Z}_{6}^{\prime}$ orbifold action since $\theta$ acts as a $\mathbb{Z}_{3}$ rotation here. On the first two-torus, however, the analogous points transform non-trivially under the $\mathbb{Z}_{6}^{\prime}$ and $\mathbb{Z}_{2}$-subgroups,

$$
T_{(1)}^{2}: \quad \theta(1)=1, \quad \theta(2)=3, \quad \theta(3)=2 .
$$

From these data, the massless closed string spectrum can be determined, see e.g. table 9 in [54] and tables 57 and 58 in [58]. More details on the construction of the type IIA string on the factorisable $T^{6} / \mathbb{Z}_{6}^{\prime}$ orbifold and the lattice orientations may be found e.g. in [54].

In the following section, we discuss how the Standard Model with hidden $U S p(6)_{h}$ is engineered on this particular orbifold background.

\subsubsection{D6-brane configuration}

D6-branes in our model are six plus one-dimensional objects along $\mathbb{R}^{1,3} \times T^{6} / \mathbb{Z}_{6}^{\prime}$, which fill the four-dimensional Minkowski space $\mathbb{R}^{1,3}$ and wrap a three-cycle along the compact space $T^{6} / \mathbb{Z}_{6}^{\prime}$. If we represent the six-torus as the product of three two-tori, $T^{6}=\otimes_{i=1}^{3} T_{(i)}^{2}$, depicted in figure 1, the three dimensions of a factorisable cycle in the six dimensional compact space are divided into one dimension on each two-torus, i.e. a product of onecycles. In the example with Standard Model spectrum plus hidden sector $U S p(6)_{h}$ of $[1$, 2] that we consider, five different stacks of supersymmetric D6-branes labelled by $a, b$, $c, d$ and $h$ satisfy the global consistency conditions of RR tadpole cancellation and Ktheory constraints. Table 1 summarises the essential aspects of the D6-brane configuration: it contains the angles that the D6-branes form with the horizontal ( $\Omega \mathcal{R}$ invariant) O6plane. On $T_{(2)}^{2}$, there is no action of the $\mathbb{Z}_{2}$ symmetry. Therefore, the D6-branes can be continuously displaced. At first instance, one can take the D6-branes to pass trough the origin, i.e. point 1 in figure 1 . On $T_{(1)}^{2}$ and $T_{(3)}^{3}$, the $\mathbb{Z}_{2}$ symmetry acts non-trivially, and the fractional D6-branes pass through $\mathbb{Z}_{2}$ invariant points. The notation $\sigma^{i}=0$ in the fourth column denotes one-cycles passing through the origin (point 1), while $\sigma^{i}=1$ denotes displacements along the lattice direction $\frac{\pi_{2 i-1}}{2}$ (to point 4 on $T_{(1)}^{2}$, point 2 on $T_{(3)}^{3}$ ), or for D6-branes wrapping the one-cycle $\pi_{2 i-1}$ displacements along $\frac{\pi_{2 i}}{2}$ (to point 5 on $T_{(1)}^{2}$, point 4 on $\left.T_{(3)}^{2}\right)$. The multiplicity and thus the rank of the gauge group of each kind of D6-branes is given in the fifth column of table 1. While the D6-branes $a, b, d$ wrapping generic three-cycles support unitary gauge factors, the stacks of D6-branes $c$ 


\begin{tabular}{|c|c|c|c|c|c|c|c|c|c|}
\hline \multicolumn{10}{|c|}{ D6-brane configuration for the Standard Model with hidden $U S p(6)_{h}$ on $T^{6} / \mathbb{Z}_{6}^{\prime}$} \\
\hline D6-brane & $\begin{array}{l}\vec{\nabla} \\
\text { త్ర } \\
\text {. }\end{array}$ & $\begin{array}{c}\text { angle on } \\
\left(T_{(1)}^{2}, T_{(2)}^{2}, T_{(3)}^{2}\right) \\
\text { w.r.t. } \Omega \mathcal{R} \text {-plane }\end{array}$ & 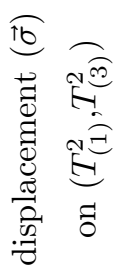 & 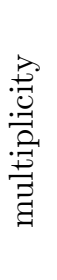 & 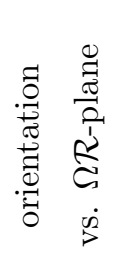 & $\begin{array}{l}\text { gauge } \\
\text { group }\end{array}$ & $\begin{array}{l}\text { wrapping } \\
\text { numbers } \\
\left(n^{i}, m^{i}\right)\end{array}$ & 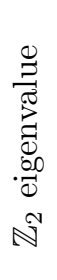 & 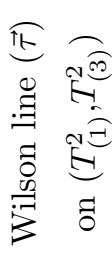 \\
\hline Baryonic & $a$ & $(-\pi / 3,-\pi / 6, \pi / 2)$ & $(1 ; 1)$ & 3 & & $U(3)_{a}$ & $(1,-1 ; 1,0 ; 0,1)$ & + & $(1,1)$ \\
\hline Left & $b$ & $(\pi / 6,-\pi / 3, \pi / 6)$ & $(1 ; 0)$ & 2 & & $U(2)_{b}$ & $(1,1 ; 2,-1 ; 1,1)$ & - & $(1,0)$ \\
\hline Right & $c$ & $(-\pi / 3, \pi / 3,0)$ & $(1 ; 1)$ & 1 & $\|$ & $U S p(2)_{c}$ & $(1,-1 ;-1,2 ; 1,0)$ & + & $(1,1)$ \\
\hline Leptonic & $d$ & $(\pi / 6, \pi / 3,-\pi / 2)$ & $(0 ; 1)$ & 1 & & $U(1)_{d}$ & $(1,1 ; 1,-2 ; 0,1)$ & + & $(1,1)$ \\
\hline Hidden & $h$ & $(-\pi / 3,-\pi / 6, \pi / 2)$ & $(0 ; 0)$ & 3 & $\perp$ & $U S p(6)_{h}$ & $(1,-1 ; 1,0 ; 0,1)$ & + & $(1,0)$ \\
\hline
\end{tabular}

Table 1: The five different stacks of D6-branes in the Standard Model on $T^{6} / \mathbb{Z}_{6}^{\prime}$ with hidden $U S p(6)$, together with their configurations on the factorised torus and the gauge groups they support. The displacements $(\vec{\sigma})$ specify the localisation of intersection points along $T_{(1)}^{2} \times T_{(3)}^{2}$.

and $h$ require some extra attention as it turns out that they are their own orientifold images, $(\theta c)^{\prime}=(\theta c)$ and $(\theta h)^{\prime}=(\theta h)$ (and also $c^{\prime}=\left(\theta^{2} c\right)$ and $h^{\prime}=\left(\theta^{2} h\right)$ ), where $(\theta c)$ is the rotated orbifold image of $c$ and $(\theta c)^{\prime}$ its orientifold image. More specifically, $(\theta c)$ is parallel to the horizontal $(\Omega \mathcal{R}$ invariant) O6-plane on all tori, and $(\theta h)$ is perpendicular to the same O6-plane along the four-torus $T_{(2)}^{2} \times T_{(3)}^{2}$ as listed in the sixth column of table 1. Therefore, the gauge groups that these D6-branes carry are enhanced to an $U S p(2)_{c}$ and an $U S p(6)_{h}$ group, respectively [2]. The full gauge group of the model is thus given by $U(3)_{a} \times U(2)_{b} \times U S p(2)_{c} \times U(1)_{d} \times U S p(6)_{h}$ in the seventh column of table 1 . The remaining thee columns contain the toroidal one-cycle wrapping numbers $\left(n^{i}, m^{i}\right)$ and various sign factors needed for the construction of the complete matter spectrum along the lines reviewed in section 2.2.1.

Remember that we can displace the D6-branes on the second torus away from the origin. If we now move $(\theta c)$ away from the $\Omega \mathcal{R}$-invariant $\mathrm{O} 6$-plane, we obtain a distinction between orbifold and orientifold image D6-branes, $c^{\prime} \neq\left(\theta^{2} c\right)$. As a result, the gauge group $U S p(2)_{c}$ breaks down to $U(1)_{c}$. More specifically, the fundamental representation (2) of $U S p(2)_{c}$ splits into the representations $(\mathbf{1})_{1}$ and $(\mathbf{1})_{-1}$ of $U(1)_{c}$. Fields with $U(1)_{c}$ charge that stem from the same $U S p(2)_{c}$ doublet still share field theoretical selection rules and formal expressions on couplings differing only in the size of the spanned worldsheets, since interaction terms are (up to the opposite variation of the size of worldsheets) invariant under the larger right-symmetric group $U S p(2)_{c}$. The Higgs-up $H_{u}$ and Higgs-down $H_{d}$ particles originate for example from an $U S p(2)_{c}$ doublet. The same is true for the right- 
handed quarks, e.g. $\left(u_{R}, d_{R}\right)$, and right-handed leptons, e.g. $\left(e_{R}, \nu_{R}^{e}\right)$. Phenomenological implications of the breaking $U S p(2)_{c} \rightarrow U(1)_{c}$ are discussed further in section 3.3.2.

The situation is different for the stack of D6-branes $h$ since its $\theta$-orbit is perpendicular to the $\Omega \mathcal{R} \theta^{-2 k}$ invariant orbit of O6-planes along $T_{(2)}^{2}$, and therefore a displacement does not alter the orientifold invariance of $h$, and $U S p(6)_{h}$ remains unbroken.

By now, the gauge group of our model is $U(3)_{a} \times U(2)_{b} \times U(1)_{c} \times U(1)_{d} \times U S p(6)_{h}$, where each $U\left(N_{x}\right)$ can be decomposed into $S U\left(N_{x}\right) \times U(1)_{x}$. We thus have four Abelian gauge groups, $U(1)_{a} \times U(1)_{b} \times U(1)_{c} \times U(1)_{d}$, which split into two anomalous massive and two anomaly-free massless Abelian gauge factors. The generalised Green-Schwarz mechanism cancels the anomalies [59] while giving masses of the order of the string scale $M_{\text {string }}$ and absorbing some axionic partners of geometric complex structure moduli in the type IIA string theory language. The two independent massless anomaly-free symmetries include the hyper charge of the Standard Model [1, 2],

$$
Q_{Y}=\frac{1}{6} Q_{a}+\frac{1}{2} Q_{c}+\frac{1}{2} Q_{d}, \quad \text { and } \quad Q_{B-L}=\frac{1}{3} Q_{a}+Q_{d}
$$

where the index $B-L$ refers to the difference between baryon and lepton number. ${ }^{3}$ In nature, the $B-L$ symmetry is not observed as a gauge symmetry, but rather as a global symmetry. There must thus be some mechanism by which these gauge bosons are rendered massive without making the hyper charge massive at the same time. There is indeed such a candidate: the chiral multiplet of the right handed neutrino contains a scalar field, the sneutrino $\tilde{\nu}_{R}$, which is a singlet under all Standard Model gauge transformations but carries a charge under the $B-L$ symmetry. If this singlet receives a nonzero vacuum expectation value (vev), it is expected to break the $B-L$ gauge symmetry. In the low energy limit, the $B-L$ symmetry then remains as a global symmetry. We conclude this section by observing that the initial gauge group $U(3)_{a} \times U(2)_{b} \times U S p(2)_{c} \times U(1)_{d} \times U S p(6)_{h}$ of the D6-brane configuration is reduced to the group $S U(3)_{a} \times S U(2)_{b} \times U(1)_{Y} \times U S p(6)_{h}\left(\times U(1)_{B-L}\right)$ which survives the transition to the low-energy field theory with $U S p(2)_{c} \rightarrow U(1)_{c}$ broken by some vev of the complex scalar in the symmetric representation, and with $U(1)_{B-L}$ rendered massive by the vev of some right-handed sneutrino $\tilde{\nu}_{R}$. The remaining low-energy gauge group is nothing but the Standard Model group times an extra 'hidden' $U S p(6)_{h}$ group.

In the following section, we present the matter spectrum and the exact localisation of each massless open string state along the compact directions.

\footnotetext{
${ }^{3}$ Note that also $Q_{c}=2 Q_{Y}-Q_{B-L}$ is anomaly free, since it stems from the breaking of $U S p(2)_{c}$ along some flat direction.
} 


\subsection{Full particle spectrum}

\subsubsection{General strategy}

The computation of the massless matter spectrum is based on inspection of the vanishing or non-vanishing of some intersection angle among D6-branes $x$ and $y$ on all three two-tori $T_{(i)}^{2}$ and on the $\mathbb{Z}_{2}$ and $\Omega \mathcal{R} \theta^{k}$ transformation properties of the intersection points and the localised massless open string states. If on the one hand an intersection point at angles is not at a $\mathbb{Z}_{2}$ invariant position along $T_{(1)}^{2} \times T_{(3)}^{2}$, there exists an image point under the $\mathbb{Z}_{2}$ symmetry, and a chiral multiplet is localised on such a pair of $\mathbb{Z}_{2}$ image points. If on the other hand the intersection point at angles is $\mathbb{Z}_{2}$ invariant, there might or might not be a chiral multiplet depending on whether the massless state is projected out by the $\mathbb{Z}_{2}$ symmetry or not. Instead of explicitly computing each massless state and determining its Chan-Paton label, the intersection numbers of the corresponding D6-branes can be used as follows, more details on the matching of methods can be found in [1] and appendix A.2 of [2]. As a starting point, the chirality due to the intersection of D6-branes $x$ and $y$ can be computed. We first calculate the intersection number on the factorisable six-torus,

$$
I_{x y} \equiv \prod_{i=1}^{3}\left(n_{x}^{i} m_{y}^{i}-m_{x}^{i} n_{y}^{i}\right),
$$

where $\left(n_{x}^{i}, m_{x}^{i}\right)$ are the wrapping numbers of D6-brane $x$ along the basic one-cycles $\pi_{2 i-1}$ and $\pi_{2 i}$ on the $i$-th two-torus $T_{(i)}^{2}$. For an ordinary six-torus, this number suffices. However, the compact space in our model is a $\mathbb{Z}_{2 N}$ orbifold of the six-torus. Therefore we also need to calculate the number $I_{x y}^{\mathbb{Z}_{2}}$ of $\mathbb{Z}_{2}$ invariant intersections among D6-branes $x$ and $y$. This number depends on the $\mathbb{Z}_{2}$ eigenvalue \pm 1 of the massless open string state at the intersection point and on the fact whether or not there is a relative Wilson line between the D6-branes $x$ and $y$, for more details see $[54,1,2]$. The intersection number of fractional D6-branes on $T^{6} / \mathbb{Z}_{2}$ and the corresponding net-chirality of bifundamental matter states is then given by

$$
\chi_{x y} \equiv-\Pi_{x}^{\mathrm{frac}} \circ \Pi_{y}^{\mathrm{frac}}=\frac{1}{2}\left(I_{x y}+I_{x y}^{\mathbb{Z}_{2}}\right) .
$$

The absolute value $\varphi_{x y}^{3 \text { angles }} \equiv\left|\chi_{x y}\right|$ gives the total number of chiral multiplets at the intersection of D6-branes $x$ and $y$, and the sign contains information on the orientation of the open strings and therefore on the chirality (or representation) of the corresponding multiplet. The total numbers $\sum_{k=0}^{2} \chi_{x\left(\theta^{k} y\right)}, \sum_{k=0}^{2} \varphi_{x\left(\theta^{k} y\right)}$ including the three $\mathbb{Z}_{3}$ orbifold images on $T^{6} / \mathbb{Z}_{6}^{\prime}$ for the Standard Model with hidden $\operatorname{USp}(6)_{h}$ were calculated in [54, 1 , 2], while in appendix A of this article we display the full set of individual contributions $\chi_{x\left(\theta^{k} y\right)}, \varphi_{x\left(\theta^{k} y\right)}$ with $k=0,1,2$ here for the first time. Also the localisation of each massless 
matter state at intersection points on $T^{6} / \mathbb{Z}_{6}^{\prime}$ is presented here for the first time. If $\chi_{x y}>0$, we use the convention that open strings are oriented from D6-brane $y$ to $x$ (which will be denoted as $[x y])$, and the associated chiral multiplets carry the representation $\left(\overline{\mathbf{N}}_{x}, \mathbf{N}_{y}\right)$, where $\mathbf{N}_{x}$ is the fundamental representation of the gauge group $U\left(N_{x}\right)$ of D6-brane stack $x$, while $\overline{\mathbf{N}}_{x}$ is the conjugate representation. If $\chi_{x y}<0$, open strings are oriented from D6-brane $x$ to $y([y x])$ and the matter fields transform in the representation $\left(\mathbf{N}_{x}, \overline{\mathbf{N}}_{y}\right)$. If $x^{\prime}$ is the orientifold image of some D6-brane $x$, then $\mathbf{N}_{x^{\prime}}=\overline{\mathbf{N}}_{x}$.

For D6-branes $x$ and $y$ coincident along the torus $T_{(2)}^{2}$ with trivial $\mathbb{Z}_{2}$ action, the charged spectrum consists of $\mathcal{N}=2$ supersymmetric non-chiral pairs of multiplets transforming as $\left(\mathbf{N}_{x}, \overline{\mathbf{N}}_{y}\right)+\left(\overline{\mathbf{N}}_{x}, \mathbf{N}_{y}\right)$ counted by the number of intersections $[1,2]$

$$
\varphi_{x y}^{\| \text {on } T_{(2)}^{2}} \equiv\left|I_{x y}^{(1 \cdot 3)}+I_{x y}^{\mathbb{Z}_{2},(1 \cdot 3)}\right| \quad \text { along } \quad T_{(1)}^{2} \times T_{(3)}^{2},
$$

where again $I_{x y}^{\mathbb{Z}_{2},(1 \cdot 3)}$ contains sign factors from relative $\mathbb{Z}_{2}$ eigenvalues and discrete Wilson lines. If the D6-branes coincide either on $T_{(1)}^{2}$ or $T_{(3)}^{2}$, the net-chirality (4) is non-vanishing. However, since there exist two states with opposite chirality and opposite $\mathbb{Z}_{2}$ eigenvalue in this sector, additional non-chiral matter pairs arise if the intersection points along $T_{(2)}^{2} \times T_{(3)}^{2}$ or $T_{(1)}^{2} \times T_{(2)}^{2}$ form $\mathbb{Z}_{2}$ pairs. The total number of representations transforming as $\left(\mathbf{N}_{x}, \overline{\mathbf{N}}_{y}\right)$ or $\left(\overline{\mathbf{N}}_{x}, \mathbf{N}_{y}\right)$ is given by $[1,2]$

$$
\varphi_{x y}^{\| \text {on } T_{(1)}^{2}} \equiv\left|I_{x y}^{(2 \cdot 3)}\right|, \quad \quad \varphi_{x y}^{\| \text {on } T_{(3)}^{2}} \equiv\left|I_{x y}^{(1 \cdot 2)}\right| .
$$

If the D6-branes are parallel but not coincident on some two-torus $T_{(i)}^{2}$, the matter spectrum is non-chiral and massive with the mass proportional to the distance of the D6-branes,

$$
M_{x y}^{2} \sim d_{(i)}^{2}(x, y)
$$

In the special case where the D6-brane $y$ is the same as $x$ or some $\mathbb{Z}_{6}^{\prime}$ orbifold image $\left(\theta^{k} x\right)$, the representation becomes $\left(\mathbf{N}_{x}, \overline{\mathbf{N}}_{x}\right)=\left(\mathbf{A d} \mathbf{j}_{x}\right)$, i.e. the adjoint representation of the gauge group $U\left(N_{x}\right)$ of D6-brane $x$. Similarly, if the D6-brane $y$ is some $\theta$-image of the orientifold image $\left(\theta^{k} x^{\prime}\right)$, the representation becomes $\left(\mathbf{N}_{x}, \overline{\mathbf{N}}_{x^{\prime}}\right)=\left(\mathbf{N}_{x}, \mathbf{N}_{x}\right)$. This representation is reducible and decomposes into a symmetric $\left(\mathbf{S y m}_{x}\right)$ and an antisymmetric $\left(\mathbf{A n t i}_{x}\right)$ representation. The existence of matter in one or both representations depends on the orientifold transformation properties of the intersection points as well as the $\Omega \mathcal{R}$ eigenvalue of the associated massless open string state, for a detailed discussion see [54, 1] and for the complementary calculation of beta function coefficients via gauge threshold amplitudes see [2, 34, 35].

The discussion of this section is general for D6-branes on orbifold in two ways: the existence of fractional D6-branes stuck at fixed points of the $\mathbb{Z}_{2}$ symmetry on $T^{6} / \mathbb{Z}_{2 N}$ and the 
corresponding formulas (4) and (6) also apply to the $T^{6} / \mathbb{Z}_{4}$ orbifold of [60] and the $T^{6} / \mathbb{Z}_{6}$ models in [61-63]. In addition, D6-branes $x$ have orbifold images $\left(\theta^{k} x\right)$ on any $T^{6} / \mathbb{Z}_{2 N}$ or $T^{6} / \mathbb{Z}_{2} \times \mathbb{Z}_{2 M}$ background with $M \neq 0$, see e.g. [64, 62, 65] for models on $T^{6} / \mathbb{Z}_{2} \times \mathbb{Z}_{4}$ and $[66,58]$ for other $T^{6} / \mathbb{Z}_{N} \times \mathbb{Z}_{M}$ orbifold backgrounds.

By the above described method, not only the complete, chiral plus vector-like, massless matter content can be derived, but also the localisation of each multiplet on $T^{6} / \mathbb{Z}_{6}^{\prime}$ can be determined, which is a necessary prerequisite for computing the size of Yukawa-like three-point interactions due to worldsheet instantons in section 3.

\subsubsection{The Standard Model on $T^{6} / \mathbb{Z}_{6}^{\prime}:$ massless states and localisations}

Let us explain the counting and localisation of matter states by the explicit example of the intersection points between the stack of D6-branes $b$ and the orbit of $\mathbb{Z}_{6}^{\prime}$ images $\left(\theta^{k} d\right)_{k=0 \ldots 2}$ of D6-brane $d$, at which the left-handed leptons $L_{i}$ are localised. The three-cycles wrapped by these D6-branes are depicted in figure 2, and labels for the intersection points are given, see also table 2.
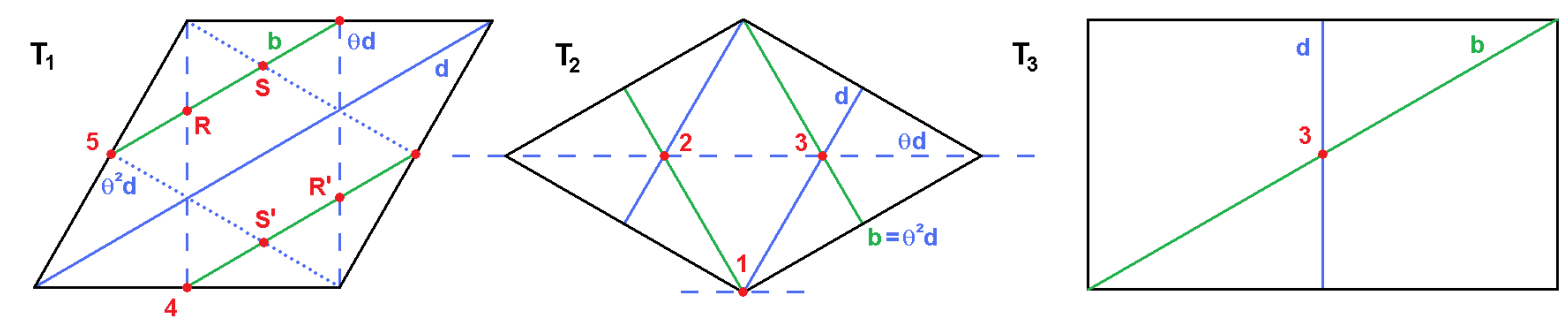

Figure 2: Configuration of the stack of D6-branes b (green) and D6-brane d (blue) with its orbifold images $\left(\theta^{k} d\right)_{k=1,2}$ (dashed and dotted blue) on the factorisable six-torus. Points $1 \ldots 6$ correspond to $\mathbb{Z}_{2}$ or $\mathbb{Z}_{3}$ orbifold fixed points, while $R \stackrel{\mathbb{Z}_{2}}{\leftrightarrow} R^{\prime}, S \stackrel{\mathbb{Z}_{2}}{\leftrightarrow} S^{\prime}$ correspond to points not fixed under the orbifold symmetry.

The interpretation is as follows. The D6-branes $b$ and $d$ do not intersect on the first torus, instead they are parallel but not coincident and do not support any massless matter state in the bifundamental representation. This conclusion agrees with $\chi_{b d}=\emptyset$ on the first line of table 2. The D6-branes $b$ and $(\theta d)$ intersect once on $T_{(3)}^{2}$, while on $T_{(2)}^{2}$ they intersect in three different points. On $T_{(1)}^{2}$, the D6-branes intersect in the three points $4, R, R^{\prime}$, where $R$ and $R^{\prime}$ are each others' image under the $\mathbb{Z}_{2}$ symmetry. Since the D6-branes $b$ and $(\theta d)$ are at angles on all three two-tori, there is one chiral multiplet localised at the points $R$ and $R^{\prime}$ together. In other words, on $T_{(1)}^{2}$ there exist two $\mathbb{Z}_{2}$ invariant orbits of intersection points localised at point 4 and the other spread over $R$ and $R^{\prime}$. Therefore, 


\begin{tabular}{|c|c|c|c|c|c|}
\hline \multicolumn{6}{|c|}{ Matter multiplicities and localisations of leptons } \\
\hline \hline Particle & $x y$ & $\chi_{x y}$ or $\varphi_{x y}$ & $T_{(1)}^{2}$ & $T_{(2)}^{2}$ & $T_{(3)}^{2}$ \\
\hline \hline \multirow{3}{*}{$L_{i, i=1 \ldots 6}$} & $b d$ & $\emptyset$ & $\| \neq$ & $1,2,3$ & 3 \\
& $b(\theta d)$ & -6 & $4,\left(R, R^{\prime}\right)$ & $1,2,3$ & 3 \\
& $b\left(\theta^{2} d\right)$ & $|4|_{m}$ & $5,\left(S, S^{\prime}\right)$ & $\|=$ & 3 \\
\hline
\end{tabular}

Table 2: Example: the total matter content $\varphi_{x y}$ for non-chiral sectors $|\cdot|$ or net-chirality $\chi_{x y}$ with sign for sectors with $\varphi_{x y}=\left|\chi_{x y}\right|$ at the intersection points between the stacks of D6-branes $b$ and $\left(\theta^{k} d\right)_{k=0 \ldots 2}$ per two-torus. The index $m$ indicates that the corresponding states stem from $\mathcal{N}=2$ sectors and acquire a mass upon relatively displacing the D6-brane stacks along $T_{(2)}^{2}$ in a continuous way. $\| \neq$ denotes parallel displaced D6-branes supporting massive string states only, and $\|=$ corresponds to coincident D6-brane positions which can support massless string states. The labels of intersection points are defined in figure 2. The complete list of massless matter localisations and their Kähler metrics for the Standard Model with hidden $U S p(6)_{h}$ on $T^{6} / \mathbb{Z}_{6}^{\prime}$ is given in table 6 of appendix A for bifundamental and table 7 for symmetric and antisymmetric matter.

in total, six $\mathbb{Z}_{2}$ invariant orbits of intersection points exist on the six-torus. This number matches the number $\left|\chi_{b(\theta d)}\right|=6$, and none of the massless states is projected out. Finally, for the D6-branes $b$ and $\left(\theta^{2} d\right)$, there exist one intersection point on $T_{(3)}^{2}$ and two on $T_{(1)}^{2}$. However, on $T_{(2)}^{2}$ the D6-branes are parallel. If we continuously displace one of the D6brane stacks on the second torus, they are still parallel but no longer coincident. The mass of the strings stretching between $b$ and $\left(\theta^{2} d\right)$ scales with the distance between the D6branes along $T_{(2)}^{2}$, cf. equation (7). The corresponding bifundamental multiplets organise themselves in massive $\mathcal{N}=2$ supersymmetric non-chiral matter pairs $(\mathbf{1}, \mathbf{2})_{-1 / 2}+(\mathbf{1}, \overline{\mathbf{2}})_{1 / 2}$ of $S U(3)_{a} \times S U(2)_{b} \times U(1)_{Y}$. There exist two such pairs counted by $\varphi_{b\left(\theta^{2} d\right)}=|4|_{m}$, where the index $m$ signals the option of switching on a mass term by a continuous relative Wilson line or displacement on $T_{(2)}^{2}$ along a flat direction in moduli space.

In a similar manner, all massless matter fields of the Standard Model with hidden $U S p(6)_{h}$ on $T^{6} / \mathbb{Z}_{6}^{\prime}$ have been localised at the intersection points of D6-branes on the compact space. The affiliations to intersection sectors are all summarised in appendix A in table 6 for bifundamental and adjoint representations and table 7 for symmetric and antisymmetric matter. The precise location of the representations can be determined along the lines described above or extracted from tables 8 to 14 (see also the explicit localisations in tables 2, 4 and 5 for the left-handed leptons, (anti)symmetrics and adjoints of $U(2)_{b}$, respectively, and [67] for the remaining Standard Model particles $Q_{i}, U_{i}, E_{i}, H_{i}$ and $\bar{L}_{i}$ ) and will be important to determine the selection rules and suppression factors of the 
perturbatively allowed Yukawa-like three-point interactions in section 3.

\subsubsection{Full spectrum of the Standard Model with hidden $U S p(6)_{h}$}

In $[1,2]$, the full massless spectrum is derived, based on the calculation of the intersection numbers $\chi_{x\left(\theta^{k} y\right)}$ and the total amount of matter states $\varphi_{x\left(\theta^{k} y\right)}$. Under the Standard Model gauge group $S U(3)_{a} \times S U(2)_{b} \times U(1)_{Y}$, the charges are given as $\left(\mathbf{R}_{S U(3)}, \mathbf{R}_{S U(2)}\right)_{Q_{Y}}$ below. The 'chiral' spectrum reads

$$
\begin{aligned}
{[C]=} & 3 \times\left[(\mathbf{3}, \mathbf{2})_{1 / 6}+(\overline{\mathbf{3}}, \mathbf{1})_{1 / 3}+(\overline{\mathbf{3}}, \mathbf{1})_{-2 / 3}+(\mathbf{1}, \mathbf{1})_{1}+(\mathbf{1}, \mathbf{1})_{0}+2 \times(\mathbf{1}, \mathbf{2})_{-1 / 2}+(\mathbf{1}, \mathbf{2})_{1 / 2}\right] \\
& +9 \times\left[(\mathbf{1}, \overline{\mathbf{2}})_{-1 / 2}+(\mathbf{1}, \overline{\mathbf{2}})_{1 / 2}\right] \\
\equiv & 3 \times\left[Q_{L}+d_{R}+u_{R}+e_{R}+\nu_{R}+2 \times L+\bar{L}\right]+9 \times\left[H_{d}+H_{u}\right]
\end{aligned}
$$

where the Higgs pairs $\left(H_{u}, H_{d}\right)$ and the lepton-anti-lepton pairs $(L, \bar{L})$ are non-chiral with respect to the Standard Model gauge group, but arise from non-vanishing intersection numbers due to the anomalous $U(1)_{b} \subset U(2)_{b}$ symmetry of the underlying D6-brane configuration.

The 'non-chiral' spectrum,

$$
\begin{aligned}
{[V]=2 \times } & \left(\mathbf{8}_{\mathrm{Adj}}, \mathbf{1}\right)_{0}+10 \times\left(\mathbf{1}, \mathbf{3}_{\mathbf{A d j}}\right)_{0}+26 \times(\mathbf{1}, \mathbf{1})_{0} \\
+ & +[\mathbf{3}, \mathbf{2})_{1 / 6}+3 \times(\overline{\mathbf{3}}, \mathbf{1})_{1 / 3}+3 \times(\overline{\mathbf{3}}, \mathbf{1})_{-2 / 3}+3 \times\left(\overline{\mathbf{3}}_{\mathrm{Anti}}, \mathbf{1}\right)_{1 / 3}+6 \times\left(\mathbf{1}, \mathbf{3}_{\mathbf{S y m}}\right)_{0} \\
& \quad+1_{m} \times(\mathbf{1}, \overline{\mathbf{2}})_{-1 / 2}+1_{m} \times(\mathbf{1}, \overline{\mathbf{2}})_{1 / 2}+2_{m} \times(\mathbf{1}, \mathbf{2})_{-1 / 2}+1_{m} \times(\mathbf{1}, \mathbf{2})_{1 / 2} \\
& \left.+4_{m} \times\left(\mathbf{1}, \mathbf{1}_{\text {Anti }}\right)_{0}+2_{m} \times(\mathbf{1}, \mathbf{1})_{1}+1_{m} \times(\mathbf{1}, \mathbf{1})_{0}+\text { c.c. }\right]
\end{aligned}
$$

consists of the (microscopically $\mathcal{N}=2$ supersymmetric) vector-like matter states on the last two lines, which acquire masses by continuous parallel relative displacements of D6branes indexed by $m$ at their multiplicities, and of the (pairs of microscopically $\mathcal{N}=1$ supersymmetric) vector-like matter states on the first two lines, which can receive masses via Yukawa-like three-point couplings as discussed in detail in section 4.

The above parts of the spectrum are uncharged under the 'hidden' gauge group $U S p(6)_{h}$. The final part of the massless open string spectrum consists of the 'hidden' sector fields that transform non-trivially under $U S p(6)_{h}$ with representations denoted as $\left(\mathbf{R}_{S U(3)}, \mathbf{R}_{S U(2)} ; \mathbf{R}_{U S p(6)}\right)_{Q_{Y}}$,

$$
[H]=2 \times\left(\mathbf{1}, \mathbf{1} ; \mathbf{1} \mathbf{5}_{\text {Anti }}\right)_{0}+(\mathbf{1}, \mathbf{2} ; \mathbf{6})_{0}+(\mathbf{1}, \overline{\mathbf{2}} ; \mathbf{6})_{0}+2 \times\left[(\mathbf{1}, \mathbf{1} ; \mathbf{6})_{1 / 2}+(\mathbf{1}, \mathbf{1} ; \mathbf{6})_{-1 / 2}\right]
$$




$$
\equiv\left[X_{1}+X_{2}\right]+\Pi^{+}+\Pi^{-}+\sum_{i=1}^{2}\left[\Omega_{i}^{+}+\Omega_{i}^{-}\right],
$$

where for later convenience of shortening the notation of three-point interactions the representations are parameterised by $X_{i, i=1,2}, \Pi^{ \pm}$and $\Omega_{i=1,2}^{ \pm}$on the last line.

The states in equations (8), (9) and (10) constitute the complete massless particle spectrum of the $T^{6} / \mathbb{Z}_{6}^{\prime}$ Standard Model with hidden sector $U S p(6)_{h}$. Apart from the three quark and lepton generations and some Higgses, it contains an abundance of vector-like fields transforming as non-chiral quark or lepton pairs or in exotic representations. We discuss in section 4 below how all of these fields can obtain masses via three-point couplings with some Standard Model-singlet fields that can acquire vers along some flat direction, similar to the Higgs field in the Standard Model Yukawa couplings.

\section{Yukawa couplings}

\subsection{Superpotential terms}

Closed triangles between D6-branes result in interactions between matter fields at the apexes due to the existence of worldsheet instantons sweeping the enclosed areas as thoroughly explained in $[47,48]$, see also $[45,46,68]$, and briefly reviewed here.

Suppose that three intersecting D6-branes $x, y$ and $z$ form a triangle on each two-torus and that each of the intersection points carries a massless string that represents a chiral matter field $\phi_{x y}^{i}, \phi_{y z}^{j}$ and $\phi_{z x}^{k}$ in the respective representation $\left(\overline{\mathbf{N}}_{x}, \mathbf{N}_{y}\right),\left(\overline{\mathbf{N}}_{y}, \mathbf{N}_{z}\right)$ and $\left(\overline{\mathbf{N}}_{z}, \mathbf{N}_{x}\right)$ of $U\left(N_{x}\right) \times U\left(N_{y}\right) \times U\left(N_{z}\right)$. This configuration gives rise to a three-point Yukawa coupling term in the superpotential,

$$
W=W_{i j k} \phi_{x y}^{i} \phi_{y z}^{j} \phi_{z x}^{k}
$$

If we take into account the orientations of the strings, i.e. if we have a sequence $[x y]+$ $[y z]+[z x]$, we are guaranteed that such a term is gauge invariant because the fields are in the bifundamental representations of the full gauge group as stated above, and the gauge transformations cancel pair-wise in the expression (11).

A purely field theoretical analysis of the matter representations would lead to allowing additional gauge invariant terms in the superpotential that are actually forbidden by the D6-brane model because there exists no closed triangle. Therefore, the D6-brane setup in type IIA string theory serves as a selection rule on allowed interaction terms once the 
localisation of each matter state in a given model is known. This is a strong restriction and goes well beyond the argument of gauge invariance as we will show below for several excluded types of couplings in the Standard Model spectrum with hidden $U S p(6)_{h}$ on fractional D6-branes in the $T^{6} / \mathbb{Z}_{6}^{\prime}$ background of $[1,2]$.

We can extract even more information from the D6-brane model. The size of the triangle formed by three D6-branes is related to the strength of the coupling [47, 48, 45, 46, 68] as follows. Denoting the area of the enclosed triangle by $\mathcal{A}_{i j k}$, which we will often give as a fraction of the total areas $\mathcal{A}_{(i)}$ of the two-tori $T_{(i)}^{2}$, the coefficient of the three-point interaction term (11) is proportional to

$$
W_{i j k} \sim e^{-\mathcal{A}_{i j k} /\left(2 \pi \alpha^{\prime}\right)} .
$$

Here $\tau_{s}=1 /\left(2 \pi \alpha^{\prime}\right)$ is the string tension. If the three D6-branes intersect exactly in one point, the area $\mathcal{A}_{i j k}$ is zero and the coefficient is of order $\mathcal{O}(1)$. The larger the area is, the smaller the coefficient and the stronger the suppression of the interaction. While the superpotential receives contributions from all possible worldsheet instantons by summing over all images on the covering space of the torus [47, 48, 45, 46, 68] leading to a formula of the type (21) stated below, in this article we focus only on the leading order and estimate the size of the holomorphic Yukawa couplings $W_{i j k}$ by the smallest possible triangles.

The physical size of the quark- and lepton-Yukawa couplings is given by the product of the holomorphic superpotential coupling $W_{i j k}$ in equation (11), which arises classically, times non-holomorphic quantum contributions involving the Kähler metrics of the corresponding matter fields [69, 70, 45, 68, 44] as briefly reviewed in the context of the present D6-brane model in section 3.4. A second potential source of Yukawa hierarchies beyond the size of triangular instantonic worldsheets is therefore given by the different expressions for the Kähler metrics.

At this point, it should be noted that additional symmetries of the string compactification, such as the $\mathbb{Z}_{2}$ eigenvalues of the massless states, might lead to further selection rules, which can only be determined by more sophisticated methods such as an explicit computation of three-point couplings on the type IIA orientifold on $T^{6} / \mathbb{Z}_{2 N}$ using vertex operators in generalisation of the recently computed two-point correlators on $T^{6} / \mathbb{Z}_{6}^{\prime}$ in appendix $\mathrm{C}$ of [49].

\subsection{Yukawa couplings for the Standard Model on $T^{6} / \mathbb{Z}_{6}^{\prime}$}

As an example, we consider the Yukawa couplings between the left- and right handed leptons and the Higgs particles in the Standard Model spectrum with hidden $U S p(6)_{h}$ 
on $T^{6} / \mathbb{Z}_{6}^{\prime}[1,2]$. For the sake of simplicity of the discussion we present here the D6-brane configuration with the right-symmetric group $U S p(2)_{c}$ and the corresponding right-handed lepton doublets $E_{i} \equiv\left(e_{R}^{i}, \nu_{R}^{i}\right)$ and Higgs-doublets $H_{i} \equiv\left(H_{u}^{i}, H_{d}^{i}\right)$. In section 3.3.2, we briefly comment on the changes in size of the areas $\mathcal{A}_{i j k}$ swept by worldsheet instantons when the right-symmetric group is broken, $U S p(2)_{c} \rightarrow U(1)_{c}$, by displacing the D6-brane $c$ away from the $\Omega \mathcal{R}$ invariant position along the two-torus $T_{(2)}^{2}$ without $\mathbb{Z}_{2}$ action, and when other D6-brane displacements are applied as well to render matter in microscopically $\mathcal{N}=2$ supersymmetric sectors in the last two lines of equation (9) massive, cf. [1, 2]. The left handed leptons $L_{i}, L_{3+i}$ with $i \in\{1,2,3\}$ labelling the localisation on $T_{(2)}^{2}$ arise at intersections of the D6-branes $b$ and $(\theta d)$ as detailed in table 2, while the right handed leptons $E_{i}$ localised at points $i \in\{1,2,3\}$ on $T_{(2)}^{2}$ stem from the intersections of $c$ with $(\theta d)$, see table 6 for a complete list of all bifundamental and adjoint matter allocations to the intersection sectors. The Higgs generations $H_{i}, H_{3+i}, H_{6+i}$ with $i \in\{1,2,3\}$ again labelling the localisation on $T_{(2)}^{2}$ are partially located at intersections of the D6-brane stack $b$ with $c$ (for $H_{i}, H_{3+i}$ ) and partly with $(\theta c)$ (for $H_{6+i}$ ). Since allowed couplings originate from closed triangles between D6-branes, we can immediately deduce that the latter three kinds of Higgs fields $H_{6+i}$ do not couple directly to the leptons. This is because the only existing closed sequence of the left, right and leptonic stacks of D6-branes is given by $[c(\theta d)]+[(\theta d) b]+[b c]$, thus ruling out the $[b(\theta c)]$ intersections. The positions of the D6-
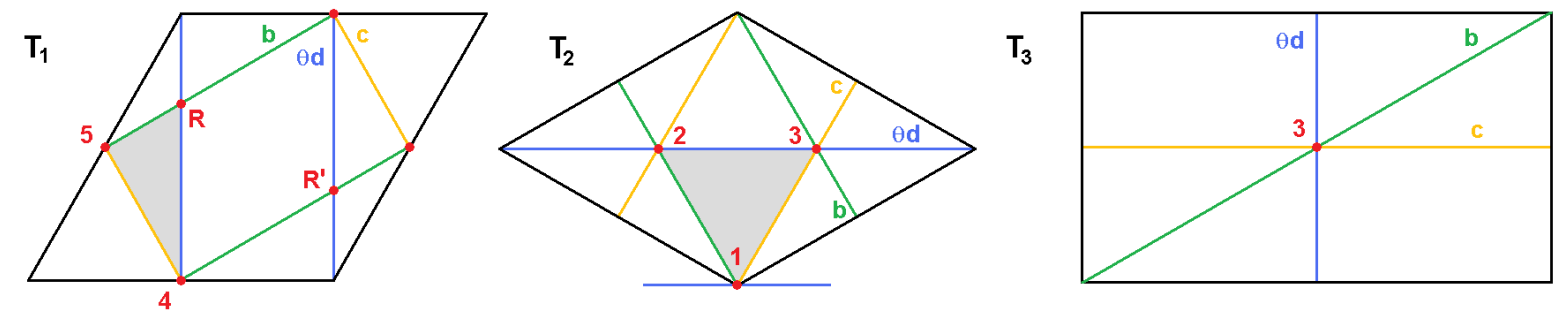

Figure 3: Position of the D6-branes $b, c$ and $(\theta d)$ and their intersection points. The shaded areas correspond to examples of instantonic worldsheets generating Yukawa interactions with flavour mixing.

brane stacks $b, c$ and $(\theta d)$ and their intersections on the factorised six-torus are displayed in figure 3. On the third torus $T_{(3)}^{2}$, all three D6-branes intersect once in the same point 3. On $T_{(1)}^{2}$, the intersection point 4 is common to the three different D6-branes. Likewise, on $T_{(2)}^{2}$, all three D6-branes intersect in point 1. Therefore, we expect an interaction term between the fields that are localised in point (413) along $T_{(1)}^{2} \times T_{(2)}^{2} \times T_{(3)}^{2}$. Since the size of the corresponding enclosed triangle is zero on each two-torus, this interaction term is not area-suppressed. Inspection of the localisation of the matter fields in table 8 of appendix A 
identifies this interaction as that of the first lepton with the first Higgs generation,

$$
W_{E_{1} L_{1} H_{1}} \sim \mathcal{O}(1)
$$

Let us now consider different points on the second two-torus $T_{(2)}^{2}$. Instead of all fields in point 1 , we choose the intersection of $(\theta d)$ with $b$ in point 2 (left-handed lepton $L_{2}$ ), the intersection of $c$ with $(\theta d)$ in point 1 (right-handed lepton pair $E_{1}$ ) and the field at the intersection of $b$ with $c$ in point 3 (Higgs pair $H_{3}$ ). These points form the closed shaded triangle on the second two-torus in figure 3 and thus result in an allowed coupling, which is suppressed compared to the previously discussed flavour diagonal one, $E_{1} L_{1} H_{1}$. By simple geometric arguments one can deduce the ratio of the area of the enclosed triangle to be $\mathcal{A}_{(2)} / 6$, where $\mathcal{A}_{(2)}$ is the total dimensionless area of $T_{(2)}^{2}{ }^{4}{ }^{4}$ Therefore we find the interaction term

$$
W_{E_{1} L_{2} H_{3}} \sim \mathcal{O}\left(e^{-\mathcal{A}_{(2)} / 6}\right) .
$$

Similarly, examining the triple intersection points 1 on $T_{(2)}^{2}$ and 3 on $T_{(3)}^{2}$, but different intersection points $4,5, R$ on $T_{(1)}^{2}$ corresponds respectively the localisations of fields $E_{1}, H_{4}$ and $L_{4}$. Their three-point interaction is also allowed by the existence of a closed triangle, and the ratio of the latter to the area of $T_{(1)}^{2}$ as displayed in figure 3 is computed as $1 / 12$,

$$
W_{E_{1} L_{4} H_{4}} \sim \mathcal{O}\left(e^{-\mathcal{A}_{(1)} / 12}\right)
$$

Finally, there exist instantonic worldsheets due to nonzero triangles on both $T_{(1)}^{2}$ and $T_{(2)}^{2}$. The example depicted in figure 3 with shaded areas on $T_{(1)}^{2} \times T_{(2)}^{2}$ corresponds e.g. to the Yukawa interaction

$$
W_{E_{1} L_{5} H_{6}} \sim \mathcal{O}\left(e^{-\mathcal{A}_{(1)} / 12} \cdot e^{-\mathcal{A}_{(2)} / 6}\right)=\mathcal{O}\left(e^{-\mathcal{A}_{(1)} / 12-\mathcal{A}_{(2)} / 6}\right)
$$

according to the labels of particle generations in the appendices. As for the examples in tables 2, 4 and 5, particle generations are labelled by their localisations on $T_{(2)}^{2}$ whenever possible.

A suppression by non-vanishing triangles along the whole six-torus does not occur at leading order for the lepton and quark Yukawas in the Standard Model example with hidden $U S p(6)_{h}$ on $T^{6} / \mathbb{Z}_{6}^{\prime}$ since the D6-branes $a, b, c, d$ pair-wise intersect once or are parallel on $T_{(3)}^{2}$. The only exception is the double intersection of stack $b$ with its orientifold image

\footnotetext{
${ }^{4}$ In the following, all two-torus areas are measured in units of $2 \pi \alpha^{\prime}$. To be in the geometric regime of the compactification, we require $\mathcal{A}_{(i)}>1$ for $i=1,2,3$. In slight abuse of the notation, we also use the symbol $v_{i} \simeq \mathcal{A}_{(i)}$ for the bulk Kähler moduli dependence of the Kähler metrics in section 3.4 and the last column of each table containing suppression factors of thee-point interactions. Last but not least, $\prod_{i=1}^{3} \mathcal{A}_{(i)} \sim \prod_{i=1}^{3} v_{i}$ denotes the volume of the $T^{6} / \mathbb{Z}_{6}^{\prime}$ orbifold, and we absorbed the numerical factors compared to the ordinary six-torus into the definition of the two-torus volumes.
} 
$b^{\prime}$ along $T_{(3)}^{2}$, which leads to three-point interactions in table 10 suppressed by $\mathcal{A}_{(3)} / 4$ of vector-like lepton or Higgs pairs with the symmetrics of $U(2)_{b}$. At sub-leading orders, i.e. performing the worldsheet instanton sum (21) over all possible copies of the defining domain of the two-tori, all three kinds of area suppressions by $\mathcal{A}_{(i)}$ with $i \in\{1,2,3\}$ will occur.

A list of all leading order lepton Yukawa couplings is given in table 8 of appendix B, and the leading quark Yukawa interactions are summarised in table 9. The appendix also lists

all allowed three-point interactions rendering the vector-like matter massive as discussed in section 4.

\subsection{Remarks on phenomenological aspects}

In the previous section, we provided a detailed discussion of the suppression of holomorphic Yukawa couplings due to triangular worldsheets for some examples of leptonic couplings in the Standard Model example on $T^{6} / \mathbb{Z}_{6}^{\prime}$. Besides only having considered the leading worldsheet contributions, generically higher order and D-instanton interactions exist. The suppression factors change upon breaking the right-symmetric group by a displacement $\sigma_{c}^{2}, U S p(2)_{c} \rightarrow U(1)_{c}$, and the existence of several Higgs fields leads to a complicated interaction pattern in the lepton and quark sectors.

\subsubsection{Higher $n$-point interactions}

Renormalisability of the supersymmetric four-dimensional field theory constrains the superpotential to maximal degree three, which corresponds to the triangular worldsheets spread among three D6-branes as discussed above for some examples of lepton couplings. An example for a renormalisable quark Yukawa coupling is given by

$$
\left[b^{\prime} a\right]+\left[a\left(\theta^{2} c\right)\right]+\left[\left(\theta^{2} c\right) b^{\prime}\right] \quad \rightarrow \quad W_{Q_{1} U_{3} H_{1}} \sim \mathcal{O}(1)
$$

and an exhaustive list of all leading quark Yukawa interactions can be found in table 9 of appendix B.

Higher order interactions may appear in two ways. In the first case, non-renormalisable couplings in perturbation theory involve more than three D6-branes. One example is given by the sequence of four D6-branes,

$$
\left[b^{\prime} a\right]+\left[a\left(\theta^{2} a\right)\right]+\left[\left(\theta^{2} a\right)\left(\theta^{2} c\right)\right]+\left[\left(\theta^{2} c\right) b^{\prime}\right] \quad \rightarrow \quad W_{Q_{1} A_{2} U_{1} H_{1}} \sim \mathcal{O}(1)
$$


in which an adjoint representation $A_{2}$ of $S U(3)_{a}$ appears. Such non-renormalisable couplings are suppressed by the cut-off scale $M_{\text {string }}$ at high energies. Since the gravitational interaction of type IIA compactifications with D6-branes on toroidal orbifolds typically requires a high string scale [71], the non-renormalisable higher $n$-point interaction terms are negligible.

The second class of interaction terms, that is not taken into account in this article, is due to instantons on wrapped Euclidean D2-branes. Their strength is suppressed by $e^{-S_{D 2}}$ and expected to be tiny in the perturbative regime of type II string theories [43]. Moreover, single instanton contributions only occur for a minimal number of fermionic zero modes. Since fractional three-cycles on $T^{6} / \mathbb{Z}_{2 N}$ backgrounds are not rigid, all single D2-instanton contributions are absent in the model on $T^{6} / \mathbb{Z}_{6}^{\prime}$.

\subsubsection{Breaking of the right-symmetric group $U S p(2)_{c} \rightarrow U(1)_{c}$}

The three-point interaction terms discussed above and listed exhaustively at leading order in appendix $\mathrm{B}$ are invariant under the unbroken right-symmetric group $U S p(2)_{c}$. For example, in the previously derived interaction $Q_{1} U_{3} H_{1}$ in equation (17), the right-handed quarks and Higgses,

$$
U_{3}=\left(\begin{array}{c}
d_{3 R} \\
-u_{3 R}
\end{array}\right) \quad \text { and } \quad H_{1}=\left(\begin{array}{c}
H_{1 u} \\
H_{1 d}
\end{array}\right),
$$

form doublets under $U S p(2)_{c}$. Upon the breaking $U S p(2)_{c} \rightarrow U(1)_{c}$ by moving the D6brane $(\theta c)$ away from the O6-plane $\left(\sigma_{c}^{2} \neq 0\right)$ along $T_{(2)}^{2}$, the interaction terms (with contraction of the $U S p(2)_{c}$ index by an epsilon tensor $\left.U_{3} H_{1} \equiv \epsilon_{i j} U_{3}^{i} H_{1}^{j}\right)$ split, e.g.

$$
W_{Q_{1} U_{3} H_{1}} Q_{1} U_{3} H_{1} \quad \longrightarrow \quad W_{Q_{1} u_{3 R} H_{1 u}} Q_{1 L} u_{3 R} H_{1 u}+W_{Q_{1} d_{3 R} H_{1 d}} Q_{1 L} d_{3 R} H_{1 d}
$$

with $W_{Q_{1} u_{3 R} H_{1 u}}=W_{Q_{1} d_{3 R} H_{1 u}} \sim \mathcal{O}\left(e^{-\Delta \mathcal{A}_{(2)}\left(\sigma_{c}^{2}\right)}\right)$ for $\sigma_{a}^{2}=\sigma_{b}^{2}=0$, where $\Delta \mathcal{A}_{(2)}\left(\sigma_{c}^{2}\right)$ denotes the size of the triangle in dependence of the displacement $\sigma_{c}^{2}$.

The vector-like matter states in the last two lines of equation (9) are all rendered massive if also the stack of D6-branes $b$ is displaced along $T_{(2)}^{2}$ with $\sigma_{b}^{2} \neq \pm \sigma_{c}^{2}$. The situation is depicted in figure 4 for the lepton Yukawa intersections of D6-branes $b, c$ and $(\theta d)$ from section 3.2, leading to modifications of the sizes of all enclosed triangles with the D6-brane stacks $b$ or $c$ along some edge. The change in size of the triangles can be computed e.g. for the case of the $[b c(\theta d)]$ sequence with displacements $0 \neq \sigma_{b}^{2} \neq \pm \sigma_{c}^{2} \neq 0, \sigma_{d}^{2}=0$, but no 


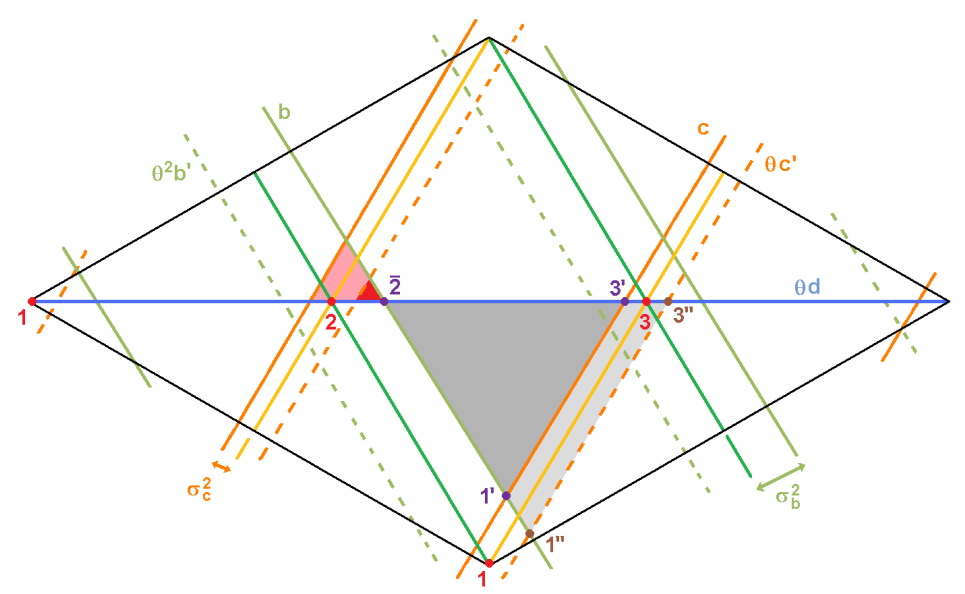

Figure 4: Change of the size of triangular worldsheets upon continuous displacements $\sigma_{b}^{2} \neq \pm \sigma_{c}^{2}$ along the $\mathbb{Z}_{2}$ invariant two-torus $T_{(2)}^{2}$. The middle lines in yellow and green correspond to the undisplaced D6-branes of figure 3, while the solid and dashed lines correspond to the D6-branes b and $\left(\theta^{2} b^{\prime}\right)$ and $c$ and $\left(\theta c^{\prime}\right)$ in green and orange, respectively.

Wilson lines $\tau_{x}^{2} \equiv 0(x=b, c, d)$ from

$$
\begin{aligned}
& \sum_{\mathcal{A}_{i j k,(2)}} e^{-\mathcal{A}_{i j k,(2)} /\left(2 \pi \alpha^{\prime}\right)} \sim \vartheta\left[\begin{array}{c}
\delta_{2} \\
0
\end{array}\right]\left(\frac{t_{2} \mathcal{A}_{(2)}}{2 \pi}\right)=\sum_{l \in \mathbb{Z}} e^{-t_{2} \mathcal{A}_{(2)}\left(\delta_{2}+l\right)^{2}} \quad \text { with } \quad t_{2}=\frac{\left|I_{b c}^{(2)} I_{c(\theta d)}^{(2)} I_{(\theta d) b}^{(2)}\right|}{\operatorname{gcd}^{2}\left(I_{b c}^{(2)}, I_{c(\theta d)}^{(2)}, I_{(\theta d) b}^{(2)}\right)}=3, \\
\delta_{2}= & \frac{i}{I_{b c}^{(2)}}+\frac{j}{I_{c(\theta d)}^{(2)}}+\frac{k}{I_{(\theta d) b}^{(2)}}+\frac{\operatorname{gcd}\left(I_{b c}^{(2)}, I_{c(\theta d)}^{(2)}, I_{(\theta d) b}^{(2)}\right)\left(I_{b c}^{(2)} \sigma_{d}^{2}+I_{c(\theta d)}^{(2)} \sigma_{b}^{2}+I_{(\theta d) b}^{(2)} \sigma_{c}^{2}\right)}{I_{b c}^{(2)} I_{c(\theta d)}^{(2)} I_{(\theta d) c}^{(2)}}+\frac{s(i, j, k)}{\operatorname{gcd}\left(I_{b c}^{(2)}, I_{c(\theta d)}^{(2)}, I_{(\theta d) b}^{(2)}\right)} \\
= & \frac{i+j+k}{3}+\frac{\sigma_{b}^{2}+\sigma_{c}^{2}}{3}+\frac{s(i, j, k)}{3}
\end{aligned}
$$

which corresponds to the Yukawa couplings in the non-coprime case [47] with $I_{b c}^{(2)}=I_{c(\theta d)}^{(2)}=I_{(\theta d) b}^{(2)}=3$. In the absence of displacements, i.e. $\sigma_{b}^{2}=\sigma_{c}^{2}=\sigma_{d}^{2}=0$, the leading term $i=j=k=0$ in the sum provides the unsuppressed Yukawa intersections for a given generation $E_{n} L_{n} H_{n}$ while for $i+j+k=3$ we recover the suppression factor of $1 / 6$ of the two-torus area $\mathcal{A}_{(2)}$ of $T_{(2)}^{2}$ for the couplings like $E_{1} L_{2} H_{3}$ in figure 3 .

With $\sigma_{b}^{2} \neq 0$ rendering all vector-like $\mathcal{N}=2$ supersymmetric matter on the last-but-one line as well as the antisymmetrics of $U(2)_{b}$ on the last line of equation (9) massive and with $\sigma_{c}^{2} \neq 0, \pm \sigma_{b}^{2}$ leading to the breaking $U S p(2)_{c} \rightarrow U(1)_{c}$ while rendering the remaining states on the last line of (9) massive, the couplings $e_{n, R} L_{n} H_{n, d}$ to the right-handed charged leptons become suppressed by a smaller area $\Delta \mathcal{A}_{(2)}\left(\sigma_{b}^{2}, \sigma_{c^{\prime}}^{2}\right)$ than those $\nu_{n, R} L_{n} H_{n, u}$ to the right-handed neutrinos with $\Delta \mathcal{A}_{(2)}\left(\sigma_{b}^{2}, \sigma_{c}^{2}\right)$. The situation is depicted in figure 4 as follows. The left-handed lepton $L_{2}$ is localised at the common apex of the two red and two grey 
triangles labelled $\overline{2}$. The right-handed electron $e_{2 R}$ and down-type Higgs $H_{2 d}$ sit at the remaining two apexes of the small dark red triangle, whereas the neutrino $\nu_{2 R}$ and up-type Higgs $H_{2 u}$ are supported at the two apexes of the bigger light triangle.

The area suppression of the non-diagonal couplings $e_{R 1} L_{2} H_{3 d}$ and $\nu_{R 1} L_{2} H_{3 u}$ changes in the opposite way, i.e. the former area is enlarged while the latter is reduced, as illustrated in figure 4 by the light and dark shaded areas with down-type Higgs $H_{3 d}$ and $e_{3 R}$ at the apexes $1^{\prime \prime}$ and $3^{\prime \prime}$ and up-type Higgs $H_{3 u}$ and $\nu_{3 R}$ at the apexes $1^{\prime}$ and $3^{\prime}$.

Assuming that the vers within one Higgs generation are of the same order, $\left\langle H_{1 u}\right\rangle \approx\left\langle H_{1 d}\right\rangle$, due to their origin from an $U S p(2)_{c}$ doublet, the hierarchy of the masses of the up-type and down-type quarks as well as of the right-handed charged leptons and neutrinos is generated solely by relative D6-brane displacements $\sigma_{b}^{2}, \sigma_{c}^{2}$ within a given particle generation.

\subsubsection{Family replication}

In the lepton sector, three particle generations appear naturally because the diagonal couplings

$$
W_{E_{1} L_{1} H_{1}}, \quad W_{E_{2} L_{2} H_{2}}, \quad W_{E_{3} L_{3} H_{3}} \sim \mathcal{O}(1),
$$

in table 8 correspond to the only non-suppressed leptonic three-point interaction terms in the superpotential before switching on continuous displacements $\sigma_{b}^{2}, \sigma_{c}^{2}$. The nonholomorphic prefactor discussed in more detail in section 3.4 is universal for all these couplings, and the mass hierarchy among the three lepton generations emerges from the choice of different vers of the first three Higgs generations, while the different Yukawa cou-

plings within one lepton family are generated by the brane displacements $\sigma_{b}^{2}, \sigma_{c}^{2}$ discussed in section 3.3.2.

There exist five types of subleading lepton Yukawa couplings with different volume sup- 
pression factors,

$$
\begin{aligned}
& W_{E_{i} L_{j} H_{k}} \sim \mathcal{O}\left(e^{-\mathcal{A}_{(2)} / 6}\right) \quad \text { with } \quad(i, j, k) \quad \text { permutations of }(1,2,3), \\
& W_{E_{i} L_{i+3} H_{i+3}} \sim \mathcal{O}\left(e^{-\mathcal{A}_{(1)} / 12}\right) \quad \text { with } \quad i=1,2,3 \text {, } \\
& W_{E_{i} L_{j} H_{k}} \sim \mathcal{O}\left(e^{-\mathcal{A}_{(1)} / 12-\mathcal{A}_{(2)} / 6}\right) \quad \text { with }\left\{\begin{array}{rr}
i=1 & (j, k)=(5,6),(6,5) \\
2 & (6,4),(4,6) \\
3 & (4,5),(5,4)
\end{array}\right. \\
& W_{E_{i} L_{i+3} H_{i}} \sim \mathcal{O}\left(e^{-\mathcal{A}_{(1)} / 3}\right) \quad \text { with } \quad i=1,2,3 \\
& W_{E_{i} L_{j} H_{k-3}} \sim \mathcal{O}\left(e^{-\mathcal{A}_{(1)} / 3-\mathcal{A}_{(2)} / 6}\right) \quad \text { with }\left\{\begin{array}{rr}
i=1 & (j, k)=(5,6),(6,5) \\
2 & (6,4),(4,6) \\
3 & (4,5),(5,4)
\end{array}\right.
\end{aligned}
$$

with the apexes of each triangle listed in table 8. The first kind leads to subleading mixings of the three generations in the lepton sector, which is flavour diagonal at leading order. The second, third, fourth and fifth type of area suppressed couplings provide mixings with the additional vector-like lepton pairs in equation (8). These vector-like lepton pairs $\left(L_{3+i}, \bar{L}_{i}\right)_{i=1,2,3}$ acquire masses through couplings to the symmetric representations $b_{i}$ of $U(2)_{b}$ as discussed below in section 4.1.1.

The situation is more complicated in the quark sector. As detailed in table 9 in appendix $\mathrm{B}$, only two right-handed quark generations $U_{1}, U_{3}$ contribute to the non-suppressed holomorphic Yukawa couplings, which involve only one of the previously considered Higgs generations, $H_{1}$, plus two other Higgs generations, where $H_{4}$ contributes to volume suppressed lepton Yukawa couplings, and $H_{7}$ does not have any three-point interaction with leptons. Moreover, the non-suppressed couplings involve the left-handed quark generations $Q_{1}, Q_{3}, Q_{4}$ but not $Q_{2}$, where $Q_{4}$ and $Q_{3}$ provide the dominant contributions to the vector-like left-handed quark pair with admixtures of $Q_{1}$ and $Q_{2}$ as discussed below in section 4.1.2. The volume suppressed quark Yukawa couplings on the other hand involve three left-handed and two right-handed quark generations, $Q_{2}, Q_{3}, Q_{4}$ and $U_{2}, U_{3}$, as well as two of the 'Standard Model' Higgs generations $\mathrm{H}_{2}, \mathrm{H}_{3}$ plus four further Higgs generations $H_{5}, H_{6}, H_{8}, H_{9}$. The three Higgs generations $H_{7}, H_{8}, H_{9}$ do not couple to the lepton sector, but only to the quark sector.

The situation in the quark sector becomes even more intricate when the three different kinds of non-holomorphic prefactors due to Kähler metrics listed in the last column of table 9 are taken into account. A thorough analysis of the quark flavour structure therefore goes well beyond the scope of this article. 


\subsection{Physical Yukawa couplings and Kähler metrics}

In section 3.1, the holomorphic three-point couplings in the superpotential were discussed in terms of areas swept by worldsheet instantons. The physical Yukawa couplings $Y_{i j k}$ in the supergravity theory depend also on the Kähler potential $\mathcal{K}$ and on the open string matter Kähler metrics $K_{x y}[69,70,45,68,44]$,

$$
Y_{i j k}=\left(K_{x y} K_{y z} K_{z x}\right)^{-1 / 2} e^{\kappa_{4}^{2} \mathcal{K} / 2} W_{i j k}
$$

While the factor $e^{\kappa_{4}^{2} \mathcal{K} / 2}$ containing the Kähler potential $\mathcal{K}$ is universal, the product of the Kähler metrics depends on each matter state and can potentially also contribute to relative suppressions of Yukawa couplings [34, 35]. The individual formal expressions of the open string Kähler metrics have been computed at leading order in [44, 36] and [33] for three non-trivial intersection angles on the six-torus and $T^{6} / \mathbb{Z}_{2} \times \mathbb{Z}_{2}$ without and with discrete torsion, respectively, and the missing cases with one or three vanishing angles on arbitrary orbifolds were added in [34,35], where it was also explicitly shown that the Kähler metrics do not depend on Wilson line or displacement moduli and have identical expressions up to the one exception of a different normalisation for adjoint matter on parallel D6branes - for all types of bulk, fractional and rigid D6-branes on the six-torus, $T^{6} / \mathbb{Z}_{2 N}$ and $T^{6} / \mathbb{Z}_{2} \times \mathbb{Z}_{2 M}$ orbifold backgrounds with discrete torsion, respectively. Recently, it was shown in [49] for the six-torus and $T^{6} / \mathbb{Z}_{6}^{\prime}$ orbifold (based on computations of two-point correlators of chiral fields on the six-torus in [72]) that the one-loop corrections to the Kähler metrics of adjoints from $\mathcal{N}=1$ supersymmetric sectors vanish, while those from $\mathcal{N}=2$ supersymmetric sectors modify the definition of the Kähler moduli. All formal expressions (at leading order) for open string Kähler metrics are displayed in table 3. The prefactor $\left(K_{x y} K_{y z} K_{z x}\right)^{-1 / 2}$ in equation (24) to the physical Yukawa coupling depends thus on the three relative intersection angles $\left(\vec{\phi}_{x y}\right)$, or some one-cycle length $\sqrt{V_{x y}^{(i)}}$ and Kähler moduli $v_{i}$ for some vanishing angle $\phi_{x y}^{(i)}=0$, all of which can in principle contribute as well to a hierarchical structure of the Yukawa couplings. In tables 8 to 14 in appendix B both suppression factors due to the size of closed triangles and different Kähler metrics are displayed in the second and last column of each table, respectively, for all possible (charged) three-point interactions. As an example, all leptonic Yukawa couplings in table 8 carry the same prefactor from the Kähler metrics with $\frac{1}{2(50)^{1 / 4}} \approx 0.188$, but differ in the size of enclosed triangles, whereas the quark Yukawa couplings in table 9 have both different factors from Kähler metrics with $\frac{1}{10^{3 / 4}} \approx 0.178$ and $\frac{1}{5 \cdot 2^{1 / 4}} \approx 0.168$ and $\frac{3^{1 / 8}}{2\left(25 \pi v_{1}\right)^{1 / 4}} \approx \frac{0.193}{v_{1}^{1 / 4}}$ and different sizes of triangular worldsheets. While the numerical factors differ, the order of magnitude is identical for these examples, except for the volume dependence $v_{1}^{-1 / 4}$ in the last case, which might lead to a significant suppression of the corresponding couplings. 


\begin{tabular}{|c||cr|r|r|}
\hline \multicolumn{5}{|c|}{ Kähler metrics and intersection angles } \\
\hline \hline$\left(\vec{\phi}_{a b}\right)$ & $(0,0,0)$ & $\left(0^{(i)}, \phi^{(j)}, \phi^{(k)}\right)$ & $\left(\phi^{(1)}, \phi^{(2)}, \phi^{(3)}\right)$ \\
\hline \multirow{3}{*}{$K_{\mathbf{R}_{a}}$} & $\frac{\sqrt{2 \pi} c_{a}}{\frac{f(S, U)}{v_{i}} \sqrt{\frac{V_{a a}^{(j)} V_{a a}^{(k)}}{V_{a a}^{(i)}}}} \quad \mathbf{A d j}_{a}$ & $f(S, U) \sqrt{\frac{2 \pi V_{a b}^{(i)}}{v_{j} v_{k}}}$ & $\frac{f(S, U)}{\sqrt{v_{1} v_{2} v_{3}}} \sqrt{\prod_{i=1}^{3}\left(\frac{\Gamma\left(\left|\phi_{a b}^{(i)}\right|\right)}{\Gamma\left(1-\left|\phi_{a b}^{(i)}\right|\right)}\right)^{-\frac{\operatorname{sgn}\left(\phi_{a b}^{(i)}\right)}{\operatorname{sgn}\left(I_{a b}\right)}}}$ \\
& $f(S, U) \sqrt{\frac{2 \pi V_{a b}^{(i)}}{v_{j} v_{k}}}$ & $\mathbf{R}_{a} \neq \mathbf{A d j}_{a}$ & \\
\hline
\end{tabular}

Table 3: Kähler metrics for open strings at arbitrary supersymmetric intersection angles. The Kähler modulus $v_{i}$ denotes the two-torus volume of $T_{(i)}^{2} . \sqrt{V_{a b}^{(i)}}$ parameterises the one-cycle volume of D6-branes parallel along $T_{(i)}^{2}$ normalised by $1 / \sqrt{v_{i}}$ such that it only contains a complex structure moduli dependence, and the factor $f(S, U)=\left(S \prod_{i=1}^{h_{21}^{\text {bulk }}} U_{i}\right)^{-\alpha / 4}$ contains the universal dependence on the four-dimensional field theoretical dilaton $S$ and bulk complex structure moduli $U_{i}$ where $h_{21}^{\text {bulk }}=1$ and $\alpha=2$ for the $T^{6} / \mathbb{Z}_{6}^{\prime}$ background of this article. The Kähler metrics for adjoint matter carry the normalisation factor $c_{a}=1,2$ on stacks of identical bulk and fractional D6-branes, respectively, whereas all other Kähler metrics are independent of the type of (bulk, fractional or rigid) D6-brane.

To firmly establish such an extra volume suppression of physical Yukawa couplings, it will, however, be necessary to derive the holomorphic worldsheet instanton contributions in equation (11) for D6-branes at one vanishing angle on $T^{6} / \mathbb{Z}_{2 N}$ from first principles and thereby exclude the possibility of cancellations in the $v_{i}$ dependence among the two factors in the physical Yukawa interactions (24).

One might speculate that the same order of magnitude of the prefactors (except for the $v_{1}^{-1 / 4}$ dependence) is due to the limited choice of supersymmetric three-cycles on $T^{6} / \mathbb{Z}_{6}^{\prime}$, which do not exceed the RR tadpole cancellation conditions, and correspondingly a small set of relative intersection angles and normalised one-cycle volumes.

\section{Masses of non-chiral representations}

According to the method described in the previous section, we can proceed to list all leading order three-point couplings of the Standard Model with hidden $U S p(6)_{h}$ on $T^{6} / \mathbb{Z}_{6}^{\prime}$. The complete list can be found in tables 8 to 14 in appendix B. While the Yukawa couplings in the lepton and quark sector have been discussed in detail in section 3.2, in this section the couplings of the vector-like matter representations and mass terms through a Higgslike mechanism involving vevs inside the (singlets in the decomposition into irreducible 
representations under $S U\left(N_{x}\right) \times U(1)_{x}$ of the) adjoint matter representations of $U(3)_{a}$ and $U(2)_{b}$ as well as inside the symmetrics of $U(2)_{b}$ are discussed.

\subsection{The vector-like fields in the particle spectrum}

The massless matter spectrum was given in section 2.2.3 with localisations at D6-brane intersections listed in appendix $A$ in tables 6 and 7 for adjoint and bifundamental and symmetric and antisymmetric representations, respectively. Besides the three Standard Model quark and lepton generations, the model contains several representations that are vector-like with respect to the Standard Model gauge group. These can be classified as follows:

1. The 'chiral' spectrum in equation (8) contains three vector-like lepton pairs at $b(\theta d)$ plus $b\left(\theta^{2} d^{\prime}\right)$ intersections as well as six plus three Higgs generations at $b c$ and $b(\theta c)$ intersections, respectively, which are counted as 'chiral' with respect to the massive anomalous $U(1)_{b} \subset U(2)_{b}$ symmetry. Leptons and Higgses are clearly distinguished by the anomaly-free $Q_{B-L}=\frac{1}{3} Q_{a}+Q_{d}$ charge. Possible mass terms for the vector-like leptons and Higgses via three-point couplings to matter in the symmetric representation of $U(2)_{b}$ are discussed below in section 4.1.1.

2. The last two lines of the 'non-chiral' spectrum in equation (9) consist of $\mathcal{N}=2$ supersymmetric hypermultiplets at D6-branes $x$ and $y$ parallel along the $\mathbb{Z}_{2}$ invariant $T_{(2)}^{2}$, and continuous relative D6-brane displacements render the full $\mathcal{N}=2$ multiplets massive, cf. equation (7), which is denoted by the lower index $m$ of the multiplicities in equation (9) and labelled by $|\cdot|_{m}$ in tables 6 and 7 . States with such a manifest supersymmetric mass term will not be further discussed in this section.

3. The first two lines of the 'non-chiral' spectrum in equation (9) and the 'hidden' spectrum in equation (10) comprise: (i) one left-handed vector-like quark pair with two different possible mass terms at leading order discussed below in section 4.1.2, (ii) three kinds of vector-like right-handed quark pairs with exotic $B-L$ charge, for which mass terms are discussed in section 4.1.3, (iii) adjoint matter of $U(3)_{a}$ and $U(2)_{b}$ as well as vector-like pairs of symmetric matter of $U(2)_{b}$, for which vevs are discussed in section 4.2 and 4.3, respectively, (iv) matter charged under the hidden gauge group $U S p(6)_{c}$ as briefly discussed in section 5 .

In the following, mass terms via three-point couplings of the vector-like representations in items 1 and 3, which originate from two distinct microscopically $\mathcal{N}=1$ supersymmetric 
sectors in conjugate representations with respect to the Standard Model gauge group, are discussed with a Standard Model singlet field, which obtains a vev.

\subsubsection{Vector-like lepton and Higgs pairs}

The 'chiral' spectrum in equation (8) contains six leptons $L_{i}=(\mathbf{1}, \mathbf{2})_{-1 / 2}$ and three antileptons $\bar{L}_{i}=(\mathbf{1}, \mathbf{2})_{1 / 2}$, which according to table 6 are localised at $b(\theta d)$ and $b\left(\theta^{2} d^{\prime}\right)$ intersections. Due to the same anomalous $U(1)_{b} \subset U(2)_{b}$ charge, the three vector-like combinations do not have perturbative three-point couplings to adjoints $B_{i}$ of $U(2)_{b}$, but instead charge selection rules allow for perturbative couplings to the conjugate symmetric representations $b_{i}=\left(\mathbf{1}, \overline{\mathbf{3}}_{\mathbf{S y m}}\right)_{0}$ of $U(2)_{b}$ in the second line of equation (9). Similarly, the anomalous $U(1)_{b}$ charge of the Higgs multiplets $H_{d}^{i}=(\mathbf{1}, \overline{\mathbf{2}})_{-1 / 2}$ and $H_{u}^{i}=(\mathbf{1}, \overline{\mathbf{2}})_{1 / 2}$ only admits three-point interactions with the symmetrics $\bar{b}_{i}=\left(\mathbf{1}, \mathbf{3}_{\mathbf{S y m}}\right)_{0}$ of $U(2)_{b}$ at the perturbative level. The localisation of all symmetric and antisymmetric matter states of $U(2)_{b}$ are displayed in table 4.

\begin{tabular}{|c||c|c|c|}
\hline \multicolumn{3}{|c|}{ Localisation of symmetric and antisymmetric matter of $U(2)_{b}$} \\
\hline \hline \multirow{2}{*}{$b\left(\theta^{k} b^{\prime}\right)$} & representation & $\begin{array}{c}\text { intersection point } \\
\left\{T_{(1)}, T_{(2)}, T_{(3)}\right\}\end{array}$ & field label \\
\hline \hline$b b^{\prime}$ & $\left(\mathbf{1}, \overline{\mathbf{3}}_{\text {Sym }}\right)_{0}$ & $\left\{\left(R, R^{\prime}\right), i, 3\right\}$ & $b_{i}$ with $i=1,2,3$ \\
& & $\left\{\left(R, R^{\prime}\right), i, 1\right\}$ & $b_{3+i}$ with $i=1,2,3$ \\
\hline$b\left(\theta b^{\prime}\right)$ & $\left(\mathbf{1}, \mathbf{3}_{\text {Sym }}\right)_{0}$ & $\{\|=, i, 3\}$ & $\bar{b}_{i}$ with $i=1,2,3$ \\
& & $\{\|=, i, 1\}$ & $\bar{b}_{3+i}$ with $i=1,2,3$ \\
\hline \multirow{2}{*}{$b\left(\theta^{2} b^{\prime}\right)$} & {$\left[\left(\mathbf{1}, \mathbf{1}_{\text {Anti }}\right)_{0}+\text { c.c. }\right]_{m}$} & $\{5, \| \neq, k\},\left\{\left(S, S^{\prime}\right), \| \neq, k\right\}$ & \\
& & with $k=1,3$ & \\
\hline
\end{tabular}

Table 4: Localisation of symmetric and antisymmetric matter at intersection points of the D6brane $b$ and the orientifold images $\left(\theta^{k} b^{\prime}\right)$. Only labels for the fields with three-point couplings to vector-like lepton or Higgs pairs are given.

Table 10 in appendix $\mathrm{B}$ shows that the first three lepton generations $L_{1}, L_{2}, L_{3}$ do not contribute to the couplings without area suppression, confirming their interpretation in section 3.3.3 as dominant contributions to the three light physical lepton generations. The 
couplings to the conjugate symmetric representations $b_{i}$ fall into two classes,

$$
\begin{aligned}
W_{\bar{L}_{i} L_{3+i}, b_{i}} & \sim \mathcal{O}(1), \\
W_{\bar{L}_{i} L_{j} b_{k}} & \sim \mathcal{O}\left(e^{-\sum_{k=1}^{3} \mu_{k} \mathcal{A}_{(k)}}\right) \quad \text { with } \quad \vec{\mu}=(0 \text { or } 1 / 6 ; 0 \text { or } 1 / 6 ; 0 \text { or } 1 / 4) \neq \overrightarrow{0}
\end{aligned}
$$

as detailed in the upper half of table 10, which means that at leading order, massive vectorlike leptons are given by the diagonal pairs $\left(\bar{L}_{i}, L_{3+i}\right)_{i=1,2,3}$ with tiny mixings among each other and with the $\left(L_{i}\right)_{i=1,2,3}$ due to the volume suppressed couplings.

The situation is similar for the six Higgs generations $H_{i}, H_{3+i}$ at $b c$ intersections with $i \in\{1,2,3\}$ labelling their localisation along $T_{(2)}^{2}$. Their three point interactions with the

symmetrics $\bar{b}_{i}$ of $U(2)_{b}$ are listed at leading order in the lower half of table 10, displaying similar suppression factors as the leptons in equation (25). The interpretation is slightly different due to the absence of 'diagonal' mass terms. However, we expect mass eigenstates to be formed by linear combinations of the six Higgs generations $H_{i}, H_{3+i}$.

For the remaining three Higgs generations $H_{6+i}$ at $b(\theta c)$ intersections, there exists no sequence involving three D6-branes. Nevertheless, couplings of the form $H_{i} a_{j} H_{k}$, with $i=$ $7,8,9 ; k=1, \ldots, 6$ and $a_{j}$ one of the (massive) antisymmetric representations of $U(2)_{b}$ are allowed by charge selection rules and closed sequences. Also couplings like $H_{i} \bar{b}_{j} \tilde{H}$ with $i=$ 7,8,9 and $\tilde{H}$ being one of the (massive) non-chiral representations like the Higgses at the intersections $b\left(\theta^{2} c\right)$ may exist. Such a term contributes terms of the form $(H \bar{b})^{2}+(\bar{b} H)^{2}+$ $(H \tilde{H})^{2}$ to the scalar potential, and therefore a vev of $\bar{b}_{i}$ could still generate masses for the Higgses. Since the three Higgs generations $H_{6+i}$ do not have lepton Yukawa couplings and only suppressed quark Yukawas, we do not further investigate their masses at this point.

In section 4.3 the conditions for vers in the symmetric representations and their effects on gauge symmetry breaking of $S U(2)_{b} \subset U(2)_{b}$ are discussed further.

\subsubsection{Left-handed vector-like quark pair}

The massless spectrum contains four left-handed quarks $Q_{i}$ at $a b^{\prime}$ and $a\left(\theta b^{\prime}\right)$ intersections and one left-handed anti-quark $\bar{Q}$ at an $a\left(\theta^{2} b^{\prime}\right)$ intersection (cf. table 6), for which a gauge invariant mass term $W=\sum_{i} m_{i} Q_{i} \bar{Q}$ exists in field theory. But such a term is in type IIA string compactifications only expected for microscopic $\mathcal{N}=2$ supersymmetric sectors. There exist, however, several three point interaction terms between the left-handed quarks $Q_{i}, \bar{Q}$ and adjoint representations $A_{j}$ of $U(3)_{a}$ and $B_{k}$ of $U(2)_{b}$ listed in table 11 of appendix B. The localisations of adjoints $B_{k}$ of $U(2)_{b}$ are given in table 5 , while $A_{1}$ off $U(3)_{a}$ stems from the $a a$ sector, and $A_{2}$ is located in the only intersection of orbifold 


\begin{tabular}{|c|c|c|c|}
\hline \multicolumn{4}{|c|}{ Localisation of adjoint matter of $U(2)_{b}$} \\
\hline$b^{\prime}\left(\theta^{k} b^{\prime}\right)$ & representation & $\begin{array}{c}\text { intersection point } \\
\left\{T_{(1)}, T_{(2)}, T_{(3)}\right\}\end{array}$ & field label \\
\hline$b^{\prime} b^{\prime}$ & $\left(\mathbf{1}, \mathbf{3}_{\mathbf{A d j}}\right)_{0}+(\mathbf{1}, \mathbf{1})_{0}$ & not localised & $B_{1}$ \\
\hline$b^{\prime}\left(\theta^{k} b^{\prime}\right)_{k=1,2}$ & $\left(\mathbf{1}, \mathbf{3}_{\mathrm{Adj}}\right)_{0}+(\mathbf{1}, \mathbf{1})_{0}$ & $\begin{array}{c}\{4, i, \|=\} \\
\left\{\left(R, R^{\prime}\right), i, \|=\right\}\end{array}$ & $\begin{array}{c}B_{1+i} \text { with } i=1,2,3 \\
B_{4+i}, B_{7+i} \text { with } i=1,2,3\end{array}$ \\
\hline
\end{tabular}

Table 5: Localisation of adjoint matter of $U(2)_{b}$ at intersection points of the D6-branes $b^{\prime}$ and its orbifold images $\left(\theta^{k} b^{\prime}\right)_{k=1,2}$. The parameterisation is chosen to fit the discussion of masses for symmetric matter in section 4.1.4.

images $a\left(\theta^{k} a\right)_{k \in\{1,2\}}$ along $T_{(1)}^{2} \times T_{(2)}^{2}$. Two couplings, $\bar{Q} Q_{4} A_{2}$ and $\bar{Q} Q_{3} B_{2}$, are not volume suppressed, which means that if the singlets (cf. the decomposition in equation (28)) inside the adjoint representations $A_{2}$ and $B_{2}$ at intersections of orbifold image D6-branes $a\left(\theta^{k} a\right)_{k \in\{1,2\}}$ and $b\left(\theta^{k} b\right)_{k \in\{1,2\}}$ receive vevs, a linear combination of $Q_{4}$ and $Q_{3}$ will form a massive quark pair with $\bar{Q}$, and only three Standard Model chiral quark generations remain massless. This statement remains true even if the volume suppressed three-point couplings in table 11 are taken into account, which lead to a mixing of the dominant contributions to the massive left-handed quark, $Q_{4}$ and $Q_{3}$, with tiny admixtures of $Q_{1}$ and $Q_{2}$. Yukawa couplings of the three massless Standard Model quark generations have already been discussed in section 3.3.3.

Details on flat directions of vers of the singlets inside the adjoint representations of $U(3)_{a}$ and $U(2)_{b}$ are discussed below in section 4.2 .

\subsubsection{Right-handed vector-like quark exotics}

The second line of the 'non-chiral' spectrum (9) contains three kinds of vector-like righthanded quark pairs with exotic $B-L$ charge labelled $Y_{1}, Y_{3}, Y_{5}$ for $(\mathbf{3}, \mathbf{1})_{-1 / 3}$ and $Y_{2}, Y_{4}, Y_{6}$ its conjugate representation at $a\left(\theta^{k} d\right)_{k \in\{0,1,2\}}$ intersections, $Z_{1}, Z_{3}, Z_{5}$ for $(\mathbf{3}, \mathbf{1})_{2 / 3}$ and $Z_{2}, Z_{4}, Z_{6}$ its conjugate representation at $a\left(\theta^{k} d^{\prime}\right)_{k \in\{0,1,2\}}$ intersections, $W_{1}, W_{3}, W_{5}$ for $\left(\overline{\mathbf{3}}_{\text {Anti }}, \mathbf{1}\right)_{1 / 3}$ and $W_{2}, W_{4}, W_{6}$ for its conjugate representation at $a\left(\theta^{k} a^{\prime}\right)_{k \in\{0,1,2\}}$ intersections. Details on the localisation in a given intersection sector can be found in table 6 for the bifundamental representations $Y_{i}, Z_{i}$ and in table 7 for the antisymmetric representations $W_{i}$.

All bifundamental representations $Y_{i}, Z_{i}$ and antisymmetrics $W_{i}$ couple to the adjoint representation $A_{2}$ of $U(3)_{a}$ at the $a\left(\theta^{k} a\right)_{k \in\{1,2\}}$ intersection with various volume suppression fac- 
tors along $T_{(1)}^{2} \times T_{(2)}^{2}$ as listed in table 12. In analogy to the example of [47], the three-point interactions involving the adjoint $A_{2}$ can be expressed, e.g. as $\sum_{i, j=1}^{3} W_{Y_{2 i-1} Y_{2 j} A_{2}} Y_{2 i-1} Y_{2 j} A_{2}$, with the coefficients $\left(W_{Y}\right)_{i j} \equiv W_{Y_{2 i-1} Y_{2 j} A_{2}}$ in the factorised form

$$
W=A \cdot X \cdot B
$$

with $A$ and $B$ diagonal $3 \times 3$ matrices and $X$ having entries 0 or 1 only, in detail

$$
\begin{array}{ll}
X_{Y}=\left(\begin{array}{lll}
0 & 0 & 1 \\
0 & 0 & 1 \\
1 & 1 & 1
\end{array}\right) & \text { and } \quad A_{Y}=B_{Y}=\operatorname{diag}\left(e^{-\mathcal{A}_{(1)} / 16}, e^{-\mathcal{A}_{(1)} / 16-\mathcal{A}_{(2)} / 4}, 1\right), \\
X_{Z}=\left(\begin{array}{lll}
1 & 1 & 1 \\
0 & 0 & 1 \\
0 & 0 & 1
\end{array}\right) & \text { and } \quad\left\{\begin{array}{l}
A_{Z}=\operatorname{diag}\left(1, e^{-\mathcal{A}_{(1)} / 16}, e^{-\mathcal{A}_{(1)} / 16-\mathcal{A}_{(2)} / 4}\right) \\
B_{Z}=\operatorname{diag}\left(e^{-\mathcal{A}_{(1)} / 16}, e^{-\mathcal{A}_{(1)} / 16-\mathcal{A}_{(2)} / 4}, 1\right)
\end{array}\right. \\
X_{W}=\left(\begin{array}{lll}
1 & 1 & 1 \\
1 & 0 & 0 \\
1 & 0 & 0
\end{array}\right) & \text { and } \quad A_{W}=B_{W}=\operatorname{diag}\left(e^{-\mathcal{A}_{(1)} / 16}, e^{\mathcal{A}_{(1)} / 16}, e^{\mathcal{A}_{(1)} / 16}\right) .
\end{array}
$$

Upon $A_{2}$ acquiring a vev, two out of three right-handed vector-like quark pairs of each kind $Y_{i}, Z_{i}, W_{i}$ are thus rendered massive simultaneously with the left-handed vector-like quark pair $\bar{Q} Q_{4}$ discussed in section 4.1.2. The third generation of each kind acquires a mass as follows: two vector-like combinations of antisymmetric representations $\left(W_{j}, W_{k}\right)_{j \in\{3,5\}, k \in\{4,6\}}$ are localised in the $a\left(\theta a^{\prime}\right)$ sector of parallel orientifold image D6-branes. In contrast to various bifundamental sectors as well as the symmetrics in the $c\left(\theta c^{\prime}\right)$ sector, a displacement $\sigma_{a}^{2} \neq 0$ from the origin does not provide a mass term, since the stack of D6-branes $a$ is perpendicular to the orbit of O6-planes along $T_{(2)}^{2}$, and $a^{\prime}$ will have the same displacement, $\sigma_{a^{\prime}}^{2}=\sigma_{a}^{2}$, while $\sigma_{x^{\prime}}^{2}=-\sigma_{x}^{2}$ for $x \in\{b, c, d\}$. This is in agreement with the fact that $U S p(6)_{h}$ with $h$ parallel to a cannot be broken by a displacement $\sigma_{h}^{2}$ [1]. To keep the number of displacements minimal while rendering $\mathcal{N}=2$ supersymmetric sectors massive, we set $\sigma_{a}^{2}=0$ throughout this article. At this point, we make the ansatz of coupling $\left(W_{j}, W_{k}\right)_{j \in\{3,5\}, k \in\{4,6\}}$ to the adjoint representation $A_{1}$ of the aa sector. This results in the three-point interactions without volume suppression on the first line of table 12. Similarly, the vector-like combinations $\left(Y_{1}, Y_{2}\right)$ and $\left(Y_{3}, Y_{4}\right)$ arise each from a pair (under $\left.\mathbb{Z}_{2}\right)$ of ad intersection points along $T_{(1)}^{2}$. The same is true for $\left(Z_{2}, Z_{3}\right)$ and $\left(Z_{4}, Z_{5}\right)$ at $a\left(\theta d^{\prime}\right)$ intersections. For each of these pairs, couplings to $A_{1}$ are allowed by the two selection rules of charge neutralness and closed triangles. These three-point couplings without volume suppression are included on the last two rows of $Y_{i}$ and on the last two lines of $Z_{i}$ couplings in 
table 12. Alternatively, the same vector-like pairs $\left(Y_{2 i-1}, Y_{2 j}\right)$ and $\left(Z_{2 i-1}, Z_{2 j}\right)$ couple to the singlet field in the 'adjoint' $D_{1}$ of $U(1)_{d}$ from the $d d$ sector without area suppression and to the $D_{2 \ldots 10}$ at $d\left(\theta^{k} d\right)_{k \in\{1,2\}}$ intersections with various suppression factors along $T_{(1)}^{2} \times T_{(2)}^{2}$. A vev of any $D_{k}$ does, however, not provide the missing mass term for the third vector-like right-handed $W_{i}$ quark generation.

All vector-like quarks on the second line of (9) are thus rendered massive by three-point couplings to two adjoints $A_{1}$ and $A_{2}$ from the $a a$ and $a\left(\theta^{k} a\right)_{k \in\{1,2\}}$ sectors, respectively, if some veus for both states $A_{1}, A_{2}$ are generated.

\subsubsection{Vector-like symmetrics of $U(2)_{b}$}

The vector-like symmetric representations of $U(2)_{b}$ are composed of states $b_{i}=\left(\mathbf{1}, \overline{\mathbf{3}}_{\text {Sym }}\right)_{0}$ at $b b^{\prime}$ and $\bar{b}_{i}=\left(\mathbf{1}, \mathbf{3}_{\text {Sym }}\right)_{0}$ at $b\left(\theta b^{\prime}\right)$ intersections as detailed in table 4 . By the selection rule of closed triangular worldsheets, they can acquire masses via couplings to the adjoints $B_{i}$ at $b\left(\theta^{k} b\right)_{k \in\{1,2\}}$ intersections listed in table 5. The complete list of leading order couplings is given in table 13 of appendix $\mathrm{B}$ with the following result. There exist two distinct sets $\left(b_{i}, \bar{b}_{j}\right)$ with $i, j \in\{1,2,3\}$ and $i, j \in\{4,5,6\}$ among which no couplings exist due to the lack of triangles on $T_{(3)}^{2}$. At leading order with no area suppression, the three-point couplings are diagonal, $i=j$, with both adjoints $B_{4+i}$ and $B_{7+i}$ of $U(2)_{b}$ located at the same intersection points. Further area suppressed couplings lead to mixings within the two distinct sets of symmetric representations. While both couplings to the adjoints $B_{4+k}$ and $B_{7+k}$ appear at our level of discussion on equal footing, it might be possible that a fully string theoretic computation leads to further selection rules e.g. taking into account the different $\mathbb{Z}_{2}$ transformations of the two massless states per intersection. Such a selection rule does not exist for the six-torus, for which the holomorphic Yukawa interactions were derived $[47,48]$, but a detailed investigation goes beyond the scope of this article.

This completes the discussion of possible mass terms of charged vector-like states in the bifundamental, antisymmetric and symmetric representation for the Standard Model with 'hidden' $U S p(6)_{h}$ on $T^{6} / \mathbb{Z}_{6}^{\prime}$. It remains to show on the one hand in sections 4.2 and 4.3 that the assumed vers of adjoint and symmetric matter representations of $U(3)_{a}$ and $U(2)_{b}$

preserve supersymmetry and gauge symmetry, and on the other hand that these vevs also provide masses for the adjoint matter states of $S U(3)_{a}$ and $S U(2)_{b}$ on the first line of (9) themselves.

Finally, in section 5 we briefly comment on the states with hidden sector charges. 


\subsection{Vacuum expectation values of adjoint representations of $U(3)_{a}$ and $U(2)_{b}$}

In section 4.1, it was argued that all vector-like quark states acquire mass terms by couplings to matter in the adjoint representation of $U(3)_{a}$, or for the left-handed vector-like quark pair also to the adjoint representation of $U(2)_{b}$.

Supersymmetry requires the vers inside these adjoint representations to satisfy both the $D$ - and $F$-term constraints, and in order to avoid a breaking of the gauge groups, the vers have to be imposed on the singlet contribution,

$$
\left(\mathbf{9}_{U(3)_{a}}\right)=\left(\mathbf{8}_{S U(3)_{a}}\right)_{0}+(\mathbf{1})_{0}, \quad\left(\mathbf{4}_{U(2)_{b}}\right)=\left(\mathbf{3}_{S U(2)_{b}}\right)_{0}+(\mathbf{1})_{0},
$$

upon the decomposition $U\left(N_{x}\right)=S U\left(N_{x}\right) \times U(1)_{x}$.

\subsubsection{Vacuum expectation values in the adjoints of $U(3)_{a}$}

Due to the reality of the adjoint representation $A$, the $D$-term contribution from such a field vanishes identically,

$$
D^{a}(A)=-g\left(\operatorname{Tr}\left(A^{\dagger} t^{a} A\right)-\operatorname{Tr}\left(A t^{a} A^{\dagger}\right)\right)=0,
$$

where $t_{i}=t_{i}^{\dagger}$ are the generators of $S U\left(N_{a}\right) \times U(1)_{a}$ with $t_{0}=\mathbf{I} / \sqrt{N_{a}}$ and $A=\sum_{i=0}^{N_{a}^{2}-1} A^{i} t_{i}$ for the adjoint representation.

While the $D$-term condition is trivially fulfilled, the $F$-term condition relates the vers of the two singlet fields pertaining to respectively the adjoint multiplets $A_{1}$ and $A_{2}$ on parallel D6-branes, $a a$, and at the intersection of orbifold images, $a\left(\theta^{k} a\right)_{k \in\{1,2\}}$, of the Standard Model with hidden $U S p(6)_{h}$ on $T^{6} / \mathbb{Z}_{6}^{\prime}$ as follows. Analogously to the string selection rules of closed polygons used in sections 3.2 and 4.1 to determine all three-point interaction terms with bifundamental, symmetric or antisymmetric matter, we make the ansatz for the general form of the superpotential terms involving only the adjoint fields $A_{1}, A_{2}$ as depicted in figure 5 ,

$$
W=\xi \operatorname{Tr}\left(A_{1}\right)+\gamma \operatorname{Tr}\left(A_{1}^{2}\right)+\frac{\mu}{3} \operatorname{Tr}\left(A_{1}^{3}\right)+\lambda \operatorname{Tr}\left(A_{2}^{2}\right)+\frac{\beta}{3} \operatorname{Tr}\left(A_{2}^{3}\right)+\alpha \operatorname{Tr}\left(A_{1} A_{2}^{2}\right),
$$

with unknown constants $\xi, \gamma, \mu, \lambda, \beta, \alpha$. The first term only receives contributions from the 

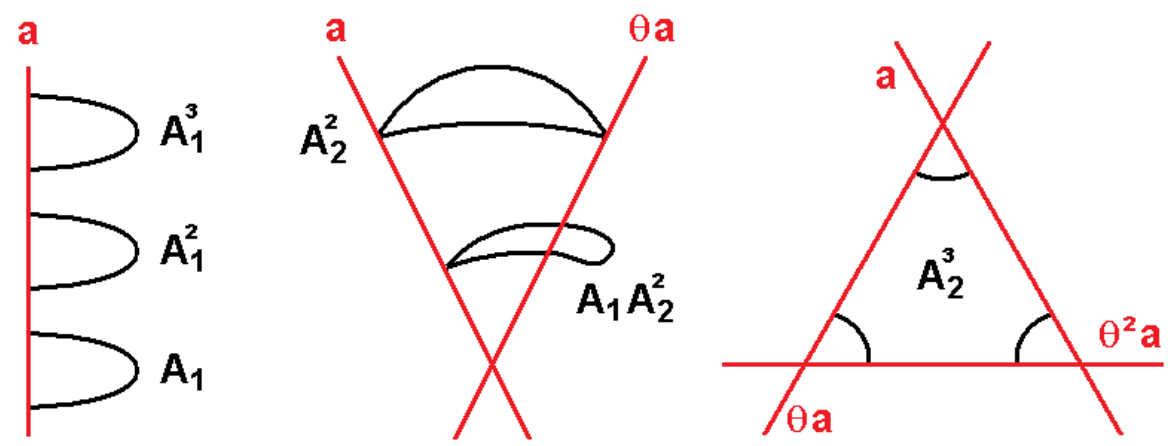

Figure 5: Intersections of the D6-branes a, with its orbifold images $(\theta a)$ and $\left(\theta^{2} a\right)$ and the couplings for the adjoint representations $A_{1}$ and $A_{2}$ of $U(3)_{a}$ which are allowed by the existence of closed polygons.

singlet inside the adjoint of $U(3)_{a} \cdot{ }^{5}$

The F-term conditions for a supersymmetric minimum in Minkowski space are given by

$$
\begin{aligned}
0 \stackrel{!}{=}-F_{1} & =\frac{\partial W}{\partial A_{1}}=\xi \mathbb{I}_{3 \times 3}+2 \gamma A_{1}^{T}+\mu A_{1}^{T} A_{1}^{T}+\alpha A_{2}^{T} A_{2}^{T}, \\
0 \stackrel{!}{=}-F_{2} & =\frac{\partial W}{\partial A_{2}}=2 \lambda A_{2}^{T}+\beta A_{2}^{T} A_{2}^{T}+\alpha\left(A_{1}^{T} A_{2}^{T}+A_{2}^{T} A_{1}^{T}\right),
\end{aligned}
$$

and with the ansatz $\left\langle A_{1}\right\rangle=a \mathbf{I}_{3 \times 3}$ and $\left\langle A_{2}\right\rangle=a b \mathbf{I}_{3 \times 3}$ with $a, b \in \mathbb{R}$ in order to preserve the $S U(3)_{a}$ gauge symmetry, or in other words only vevs for the singlet fields in equation (28), the $F$-term conditions reduce to

$$
0=\xi+2 \gamma a+\left(\mu+\alpha b^{2}\right) a^{2} \quad \text { and } \quad 0=2 \lambda a b+(\beta b+2 \alpha) a^{2} b .
$$

For $\xi=0$, the $F$-term conditions (33) have three types of solutions:

5 Strictly speaking, each coefficient depends on (untwisted) closed string moduli, just like the threepoint interactions discussed before. Throughout the section we assume that the relevant moduli take finite constant values.

In $[44,5]$ based on earlier work of the heterotic string [70], it was argued that $\xi=0$ in perturbation theory due to the existence of a conserved Abelian worldsheet current $U(1)_{W S}$, which serves as a third selection rule. Moreover, following arguments in [47] the couplings $\xi, \gamma, \mu$ involving the field $A_{1}$ only are constrained by its origin from an $\mathcal{N}=2$ vector multiplet. For vanishing superpotential contributions to $A_{1}$, i.e. $\xi=\gamma=\mu=\alpha=0$, the first F-term condition (31) is trivially fulfilled for arbitrary $\left\langle A_{1}\right\rangle$, whereas the second F-term condition (32) has the non-trivial solution $\left\langle A_{2}\right\rangle=-2 \lambda / \beta$. Such a vev recombines D6-branes $a$ with their orbifold images $\left(\theta^{k} a\right)$ thereby generating field theoretical couplings, e.g. to $A_{1}$ or quarks, that were previously forbidden by stringy selection rules. Moreover, it was argued in [73, 39] that a Polonyi term $(\xi \neq 0)$ might be generated by D2-brane instantons. We therefore present the field theoretical discussion of the superpotential in this section in a general form including several types of $A_{1}$ couplings. 
1. Both fields $A_{1}, A_{2}$ have zero vevs, i.e. $a=0$. In this case, all right-handed vector-like quarks remain massless.

2. $A_{1}$ receives a non-trivial vev, $a=-\frac{2 \gamma}{\mu}$, while $A_{2}$ remains trivial, i.e. $b=0$. In this case, one vector-like combination of right-handed quarks of each kind $W_{i}, Y_{i}, Z_{i}$ acquires a mass, while all others remain massless.

3. Both fields $A_{1}$ and $A_{2}$ receive supersymmetry preserving vevs,

$$
a=\frac{-2 \gamma}{\mu+\alpha b^{2}}=\frac{-2 \lambda}{\beta b+2 \alpha} \quad \text { and } \quad b=\frac{1}{2 \lambda \alpha}\left(3 \gamma \beta \pm \sqrt{\gamma^{2} \beta^{2}-4 \lambda \alpha(\mu \lambda-2 \alpha \gamma)}\right) .
$$

In this case, all vector-like left- and right-handed quarks acquire masses.

For $\xi \neq 0$, two solutions can be distinguished:

1. $A_{2}$ does not receive a vev, but $A_{1}$ does with $a=\frac{1}{\mu}\left(-\gamma \pm \sqrt{\gamma^{2}-\mu \xi}\right)$. Again, only one vector-like combination of each kind $W_{i}, Y_{i}, Z_{i}$ acquires a mass, while all other right-handed vector-like quark pairs remain massless.

2. Both $A_{1}$ and $A_{2}$ receive vers,

$$
a=\frac{-2 \lambda}{\beta b+2 \alpha} \quad \text { and } \quad b=\frac{2 \beta(\xi \alpha-\lambda \gamma) \pm 2 \lambda \sqrt{\gamma^{2} \beta^{2}-4 \lambda \alpha(\mu \lambda-2 \alpha \gamma)-\xi\left(4 \alpha^{3}+\beta^{2} \mu\right)}}{\xi \beta^{2}+4 \alpha \lambda^{2}} \text {. }
$$

In this case, all right- and left-handed vector-like quark pairs receive masses.

Since the superpotential (30) contains cubic terms, a non-trivial vev of the singlet term inside the adjoint $A_{1}$ of $U(3)_{a}$ renders the adjoint multiplet $\tilde{A}_{1}=\left(\mathbf{8}_{\mathbf{A d j}}, \mathbf{1}\right)_{0}$ of $S U(3)_{a} \subset$ $U(3)_{a}$ massive for $\mu \neq 0$. More explicitly, if we insert $A_{1}=a \mathbf{I}_{3 \times 3}+\tilde{A}_{1}$ and $A_{2}=a b \mathbf{I}_{3 \times 3}+\tilde{A}_{2}$ in the superpotential (30), we find

$$
W=(\gamma+\mu a) \operatorname{Tr}\left(\tilde{A}_{1}^{2}\right)+(\lambda+\alpha a+\beta a b) \operatorname{Tr}\left(\tilde{A}_{2}^{2}\right)+2 \alpha a b \operatorname{Tr}\left(\tilde{A}_{1} \tilde{A}_{2}\right)+\ldots
$$

The mass-terms for $\tilde{A}_{1}$ and $\tilde{A}_{2}$ are thus given by the mass matrix

$$
M=\left(\begin{array}{cc}
\gamma+\mu a & \alpha a b \\
\alpha a b & \lambda+\alpha a+\beta a b
\end{array}\right)=\left(\begin{array}{cc}
\gamma+\mu a & \alpha a b \\
\alpha a b & \frac{1}{2} \beta a b
\end{array}\right)
$$

where in the last equality, the $F$-term condition (33) was used. Both adjoints $\tilde{A}_{i}$ of $S U(3)_{a}$ on the first line of the 'non-chiral' spectrum in equation (9) are therefore expected to receive mass terms for $a b \neq 0$ unless the prefactors $\alpha, \beta \ldots$ in the superpotential take very special values, which could only be determined in an elaborate string theoretic computation of $n$-point correlators on the $T^{6} / \mathbb{Z}_{6}^{\prime}$ orbifold. 


\subsubsection{Comments on vacuum expectation values in the adjoints of $U(2)_{b}$}

The 'non-chiral' spectrum in equation (9) contains ten multiplets in the adjoint representation $B_{i}$ of $U(2)_{b}$, for which a complete list of localisations is given in table 5 . The computation of mass terms for the adjoints $\tilde{B}_{i}=\left(\mathbf{1}, \mathbf{3}_{\text {Adj }}\right)_{0}$ of $S U(2)_{b}$ under the decomposition in equation (28) along $D$ - and $F$-flat directions is completely analogous to the one for the adjoints $\tilde{A}_{i}$ of $S U(3)_{a}$ in section 4.2 .1 with vers for the singlets inside $B_{i}$. The existence of nine adjoint representations $B_{i, i \in\{2 \ldots 10\}}$ at $b\left(\theta^{k} b\right)_{k=1,2}$ intersections leads to a non-trivial pattern of diagonal interaction terms at one point on the six-torus plus area suppressed mixings of three types (with $i \in\{1,2,3\}$ ),

$$
\begin{aligned}
W_{\left(B_{1+i}\right)^{m}}, \quad W_{\left(B_{4+i}\right)^{n}\left(B_{7+i}\right)^{p}} & \sim \mathcal{O}(1) \quad \text { for } \quad m, n+p=2,3, \\
W_{B_{2} B_{3} B_{4}}, \quad W_{B_{5+3 m_{1}} B_{6+3 m_{2}} B_{7+3 m_{3}}} & \sim \mathcal{O}\left(e^{-\mathcal{A}_{(2)} / 6}\right) \quad \text { for } \quad m_{1}, m_{2}, m_{3} \in\{0,1\}, \\
W_{B_{1+i}\left(B_{4+i}\right)^{m}\left(B_{7+i}\right)^{2-m}} & \sim \mathcal{O}\left(e^{-\mathcal{A}_{(1)} / 24}\right) \quad \text { for } \quad m \in\{0,1,2\}, \\
W_{B_{1+i} B_{4+3 m_{1}+j} B_{4+3 m_{2}+k}} & \sim \mathcal{O}\left(e^{-\mathcal{A}_{(1)} / 24-\mathcal{A}_{(2)} / 6}\right) \quad \text { for } \quad m_{1}, m_{2} \in\{0,1\}, \\
& \text { and } \quad(i, j, k) \text { cyclic permutations of }(1,2,3) .
\end{aligned}
$$

In addition, all kinds of couplings of the adjoint $B_{1}$ from the $b b$ sector, $\operatorname{Tr}\left(B_{1}^{k}\right)_{k \in\{1,2,3\}}$ and $\operatorname{Tr}\left(B_{1} B_{1+i}^{2}\right)$ and $\operatorname{Tr}\left(B_{1}\left(B_{4+i}\right)^{n}\left(B_{7+i}\right)^{2-n}\right)_{n \in\{0,1,2\}}$, contribute to the superpotential without area suppression in analogy to equation (5). This rich structure is expected to be able to provide supersymmetric mass terms for all ten adjoints $\tilde{B}_{i}=\left(\mathbf{1}, \mathbf{3}_{\mathbf{A d j}}\right)_{0}$ of $S U(2)_{b}$ on the first line of the 'non-chiral' spectrum in equation (9) simultaneously to those of the symmetrics $\left(b_{i}, \bar{b}_{i}\right)$ discussed in section 4.1.4 with leading couplings to the $B_{i}$ listed in table 13 of appendix B. As for the adjoints of $S U(3)_{a}$, a more precise discussion of the mass terms requires the derivation of the correct prefactors in the superpotential via sophisticated string theoretic methods such as $n$-point correlation functions on the $T^{6} / \mathbb{Z}_{6}^{\prime}$ background.

\subsection{Vacuum expectation values of symmetric representations of $U(2)_{b}$}

The symmetric representation $S$ of $U(N)$ transforms as $S^{\prime}=U S U^{T}$ under gauge transformations, and by considering infinitesimal transformations $U=\mathbb{I}_{N \times N}+i \alpha$ with $\alpha \equiv \sum \alpha_{a} t^{a}$, where $t^{a}$ are the generators of $U(N)$, we can derive the generators $T_{[i j][k l]}^{a}=t_{i k}^{a} \delta_{j l}+t_{j l}^{a} \delta_{i k}$ of the symmetric representation. This leads to the $D$-term

$$
D^{a}=-g S_{[i j]} T_{[i j][k l]}^{a} S_{[k l]}^{*}=-2 g \operatorname{Tr}\left(S^{\dagger} t^{a} S\right) .
$$


Using

$$
\sum_{a} t_{i j}^{a} t_{k l}^{a}=\frac{1}{2}\left(\delta_{i l} \delta_{j k}-\frac{1}{N} \delta_{i j} \delta_{k l}\right) \quad \text { for } \quad S U(N)
$$

the contribution of a symmetric representation to the $D$-terms scalar potential is reduced to

$$
V_{D}=\frac{1}{2} \sum_{a} D^{a} D^{a}=g^{2}\left(\operatorname{Tr}\left(S^{\dagger} S S^{\dagger} S\right)-\frac{1}{2} \operatorname{Tr}\left(S^{\dagger} S\right) \operatorname{Tr}\left(S^{\dagger} S\right)\right) \geq 0 \quad \text { for } S U(2) .
$$

A flat direction, $V_{D}=0$ of this $D$-term contribution is given by the parameterisation

$$
S=e^{i \alpha}\left(\begin{array}{cc}
m & i q \\
i q & m
\end{array}\right) \quad \Rightarrow \quad S^{\dagger} S=\left(m^{2}+q^{2}\right) \mathbf{I}_{2 \times 2},
$$

for which also $D^{a}=0$, as can be explicitly computed using the Pauli matrices for the generators $t^{a}$ of $S U(2)_{b}$.

At this point it is important to notice, that for the full group $U(2)_{b}$ equation (40) is replaced by $\sum_{a} t_{i j}^{a} t_{k l}^{a}=\frac{1}{2} \delta_{i l} \delta_{j k}$, and a massless $U(1)_{b} \subset U(2)_{b}$ does not allow for flat directions, $V_{D}=g^{2} \operatorname{Tr}\left(S^{\dagger} S S^{\dagger} S\right)>0$ for $S \neq 0$. However, the Abelian group $U(1)_{b}$ in the Standard Model with hidden $U S p(6)_{h}$ on $T^{6} / \mathbb{Z}_{6}^{\prime}$ is anomalous and acquires a string scale mass via the generalised Green-Schwarz mechanism justifying our ansatz for the $D$-terms of $S U(2)_{b}$ only.

In section 4.1.1, we argued that three symmetric representations $\bar{b}_{i}$ are needed to render all vector-like lepton pairs massive. Assuming vevs of the form (42) for each representation clearly corresponds to a flat direction, $V_{D}=0$, since each contribution to the $D$-terms, $D^{a}=-2 g \sum_{i} \operatorname{Tr}\left(S_{i}^{\dagger} t^{a} S_{i}\right)=0$, vanishes.

Similar to the discussion of vevs for the adjoints $B_{i}$ of $U(2)_{b}$, the $F$-term contributions from the symmetric representations contain all possible interaction terms. Since the prefactors of the couplings are not known, we assume that a non-trivial vacuum with vevs of some symmetric representations of $U(2)_{b}$ exists. In contrast to the discussion of vevs for adjoints of $U(2)_{b}$ in section 4.2 , the symmetric representation is, however, irreducible and any vev breaks the $S U(2)_{b}$ gauge symmetry, as can be seen for example from the covariant derivative,

$$
D_{\mu} S=\partial_{\mu} S-i g\left(W_{\mu} S+S W_{\mu}^{T}\right)
$$

where $W_{\mu}$ is the gauge potential of $S U(2)_{b}$. For example, $\langle S\rangle=\left(\begin{array}{ll}0 & \mu \\ \mu & 0\end{array}\right)$ generates the mass term $8 g^{2}|\mu|^{2} W_{\mu}^{+} W_{\mu}^{-}$with $W_{\mu}^{ \pm}=\frac{1}{2}\left(W_{\mu}^{1} \pm i W_{\mu}^{2}\right)$ the standard definition of the 
charged vector-bosons of the weak gauge symmetry. In contrast to the Higgs mechanism $S U(2) \times U(1)_{Y} \rightarrow U(1)_{\text {el-mag }}$ of the Standard Model, only two out of three gauge bosons are rendered massive by the choice of some vers inside symmetric representations. We therefore postulate a step-wise breaking down to the electromagnetic gauge symmetry,

$$
S U(2)_{b} \times U(1)_{Y} \quad \stackrel{\text { at } M_{\mathrm{sym}}<M_{\text {string }}}{\longrightarrow} \quad U(1)_{I_{3}} \times U(1)_{Y} \quad \stackrel{\text { at } M_{\mathrm{el}-\mathrm{mag}}<M_{\mathrm{sym}}}{\longrightarrow} \quad U(1)_{\mathrm{el}-\mathrm{mag}},
$$

with an intermediary scale $M_{\text {sym }}$ below which the third component of the weak isospin times the hyper charge, $U(1)_{I_{3}} \times U(1)_{Y}$, remain massless. Such a mechanism is expected to affect the Weinberg angle $\theta_{W}$, which for our model is given at $M_{\text {string }}$ by $\sin ^{2} \theta_{W}=0.65$ [2]. Since the angle receives quantum corrections which depend on the energy scale, matching data at the electro-weak scale requires an in-depth study of all participating mass scales. This goes clearly beyond the scope of this article.

The above discussion focussed on the symmetric representations of $U(2)_{b}$ which occur in the model. A different way of realising that the $D$-term conditions (39) are trivially fulfilled and the $S U(2)_{b}$ symmetry broken by an arbitrary vev relies on the fact that $S U(2)$ has exactly one three-dimensional representation, and therefore the symmetric representation is equivalent to the adjoint representation.

In related models, it is possible that antisymmetric representations $\left(\mathbf{1}, \mathbf{1}_{\mathrm{Anti}}\right)_{0}$ instead of symmetric matter of $U(2)_{b}$ couple to the vector-like lepton or Higgs pairs. In this case, it is again important for the $D$-term condition that $U(1)_{b}$ is anomalous and massive, but any $v e v$ will preserve the $S U(2)_{b} \subset U(2)_{b}$ gauge symmetry.

\section{Couplings to the 'hidden' $U S p(6)_{h}$ sector}

Up to now, the discussion has focussed on supersymmetric mass terms for vector-like multiplets with only Standard Model charges. The states with hidden sector charges in equation (10) consist of antisymmetric matter $X_{1}, X_{2}$ of $U S p(6)_{h}$ coupling only gravitationally to the Standard Model plus vector-like pairs $\Pi^{ \pm}$and $\Omega_{i}^{ \pm}$with $S U(2)_{b}$ or $U(1)_{Y}$ charge, respectively, but no gauge interaction with $S U(3)_{a} \times U(1)_{B-L}$.

$U S p(2 N)$ gauge groups are good candidates for supersymmetry breaking via gaugino condensation in a strongly coupled phase. To reach the strong coupling regime at energies below $M_{\text {string }}$, the charged matter states must acquire masses. Analogously to the pairs of microscopic $\mathcal{N}=1$ supersymmetric sectors in sections 4.1.1 to 4.1.4, the 'hidden' sector fields with $S U(2)_{b}$ or $U(1)_{Y}$ charge have (at the level of triangular worldsheets) area suppressed three-point interactions with the antisymmetric representation $X_{2}$ of $U S p(6)_{h}$ 
at $h\left(\theta^{k} h\right)_{k=1,2}$ intersections listed on the last two lines of table 14 in appendix B. Assuming that the discussion for a vev of $X_{2}$ proceeds analogous to the discussion of the (anti)symmetrics of $U(2)_{b}$ in section 4.3 , all 'hidden' sector fields are rendered massive.

The 'hidden' sector matter fields are of interest also from a different point of view. Supposing that supersymmetry breaking is realised in the hidden sector, the fields with charges under $S U(2)_{b} \times U(1)_{Y}$ and $U S p(6)_{h}$ might act as messenger fields, which transmit the supersymmetry breaking to the visible sector. In that sense, also the area-suppressed couplings $\Omega_{2} \Pi^{+} H_{6+i}$ with $i \in\{1,2,3\}$ on the first lines of table 14 are of interest since in particular $i=1$ corresponds to the Higgs multiplet $H_{7}$ which participates in the non-suppressed quark Yukawa interactions of section 4.1.2 and table 9 of appendix B.

\section{Conclusions and Outlook}

We exhaustively investigated the perturbative leading order three-point interactions of matter states in the Standard Model with hidden $U S p(6)_{h}$ on intersecting D6-branes on $T^{6} / \mathbb{Z}_{6}^{\prime}$ from $[1,2]$. For the lepton Yukawa interactions, we found that the dominant terms are flavour diagonal and involve one Higgs generation per lepton family, whereas the quark sector contains even at the leading order mixings and two further Higgs generations. It will be interesting to perform a more detailed investigation of the rich flavour structure and Yukawa hierarchies of both the lepton and quark sector for this model in the future.

Based on the same selection rules of charge cancellation and on the existence of closed triangular worldsheets, we further found that (nearly) all vector-like charged states in the open string spectrum receive masses through Higgs-like couplings involving some fields with vers:

- two displacements $\sigma_{b}^{2}, \sigma_{c}^{2}$ render all vector-like exotic leptons on the last two lines of (9) massive while also creating Yukawa hierarchies within a given particle generation,

- two veus for the singlets inside the adjoints $A_{1}, A_{2}$ of $U(3)_{a}$ render all vector-like quarks on the second line of (9) massive, while also providing mass terms for the two adjoints of $S U(3)_{a}$ on the first line of equation (9),

- the vector-like symmetric representations of $S U(2)_{b}$ on the second line of (9) and the adjoints of $S U(2)_{b}$ on the first line of (9) receive masses via couplings to adjoints of $U(2)_{b}$, where vevs of (at least) three singlets are needed, 
- the 'hidden' sector fields with $S U(2)_{b} \times U(1)_{Y}$ charge acquire masses if one antisymmetric representation $X_{2}$ of $U S p(6)_{h}$ acquires a vev,

- the vector-like lepton pairs and six of the nine Higgs generations in (8) receive masses via perturbative three-point couplings to six symmetric representations of $S U(2)_{b}$, for which a vev breaks the gauge group to the third component of the weak isopsin, $U(1)_{I_{3}}$. The remaining three Higgs generations may acquire masses when also couplings to massive matter states are taken into account.

In summary, (at least) $2+2+3+1=8$ vevs are needed to provide masses for all non-chiral states with Standard Model charges in equations (9) and (10). The vector-like lepton pairs and (most) Higgs generations in equation (8) acquire at the level of perturbative three-point interactions masses if six further vevs in the symmetric and its conjugate representation of $U(2)_{b}$ are switched on. The latter will, however break $S U(2)_{b} \rightarrow U(1)_{I_{3}}$, and a thorough discussion of step-wise breaking the gauge symmetry down to the electromagnetic group at the electro-weak scale needs to be performed in the future. Taking into account non-renormalisable higher order couplings in the superpotential, which include neutral closed string fields as well, might reduce the number of required vevs while simultaneously addressing the question of moduli stabilisation.

The Standard Model sector on D6-branes $a, b, c, d$ for the model with hidden $\widehat{U S p(2})_{\hat{h}}$ in $[1,2]$ coincides with the one with hidden $U S p(6)_{h}$ discussed here, therefore all Yukawa couplings and mass terms - except for the ones involving hidden sector fields in section 5 presented in this article are identical for both known D6-brane examples of the Standard Model on $T^{6} / \mathbb{Z}_{6}^{\prime}$ with some hidden sector.

The present result of $8+6$ vevs when considering only perturbative three-point interactions can be compared to known results for the Standard Model on heterotic orbifolds such as on $T^{6} / \mathbb{Z}_{6}^{\prime}$ in [13], where non-renormalisable couplings up to the sixth order in the Standard Model singlet fields with vers were taken into account. Also in the heterotic $T^{6} / \mathbb{Z}_{2} \times \mathbb{Z}_{2}$ case of [74], a larger number, 44, of vevs was required to render the vector-like matter states massive. This may be related to the fact that we did not discuss masses for singlet fields such as the $h_{21}$ complex structure and $h_{11}^{-}$Kähler moduli which arise in the closed string sector. In heterotic orbifolds, the geometric moduli at fixed points receive charges under the gauge groups and lead to enhancements of the representations. They are therefore necessarily included in any discussion of charged vector-like states within orbifold compactifications of the heterotic string.

The investigation in this article relies on two basic selection rules of the open string perturbation theory, namely charge cancellation including the anomalous Abelian factors and 
the existence of closed triangles. In a fully string theoretic computation, these selection rules might have to be supplemented by additional 'intrinsic' symmetry properties such as the underlying $\mathbb{Z}_{2}$ orbifold symmetry. To finally decide on the existence of $n$-point interactions and determine their strength, it will be necessary to generalise the derivation of Yukawa couplings on the six-torus to $T^{6} / \mathbb{Z}_{2 N}$ and $T^{6} / \mathbb{Z}_{2} \times \mathbb{Z}_{2 M}$ orbifolds e.g. using correlators along the lines of [75-78] and the recent computation of two-point functions on $T^{6} / \mathbb{Z}_{6}^{\prime}$ in [49]. Such a computation will involve the complete worldsheet instanton sum in contrast to the leading terms with the smallest possible triangular worldsheets considered here, and it will settle the open question if the suppression factor by $1 / v_{i}^{1 / 4}$ for D6-branes parallel along $T_{(i)}^{2}$ - for which to our knowledge there exist no results in the literature - is of physical relevance to the Yukawa couplings, or if it cancels among the non-holomorphic prefactor and the holomorphic worldsheet instanton sum.

Last but not least, the non-holomorphic Kähler metrics which contribute to the prefactor of the physical Yukawa interactions are only known classically and can in principle receive corrections at any order in perturbation theory and by all kinds of non-perturbative effects. It will be interesting to see if they are indeed protected by 'intrinsic' string theoretic symmetries as suggested recently in [49].

\section{Acknowledgements}

G.H. thanks F. Saueressig for discussions and F. Marchesano for useful email correspondence. J.V. thanks W. Troost for discussions and advice.

The work of G. H. is partially supported by the "Research Center Elementary Forces and Mathematical Foundations" (EMG) at the Johannes Gutenberg-Universität Mainz. The research of J.V. has been supported in part by the Belgian Federal Science Policy Office through the Interuniversity Attraction Pole IAP VI/11 and by FWO-Vlaanderen through project G011410N.

J.V. acknowledges the support of the department of physics of the KULeuven and the kind hospitality at JGU Mainz at various stages of this project, which is partly based on the master thesis [79]. 


\section{A Tables with localisations of massless matter states}

This appendix displays for the first time all sector-wise localisations of matter states of the Standard Model with hidden $U S p(6)_{h}$ on $T^{6} / \mathbb{Z}_{6}^{\prime}$, for which the matter spectrum had been computed in $[1,2]$. The corresponding complete Kähler metrics are also given in tables 6 and 7, where those for strings with an endpoint on the stacks $a$ or $d$ have been already been computed in [34]. The positions of the intersection points in each sector are given by the apexes in the tables of three-point functions in appendix B. 


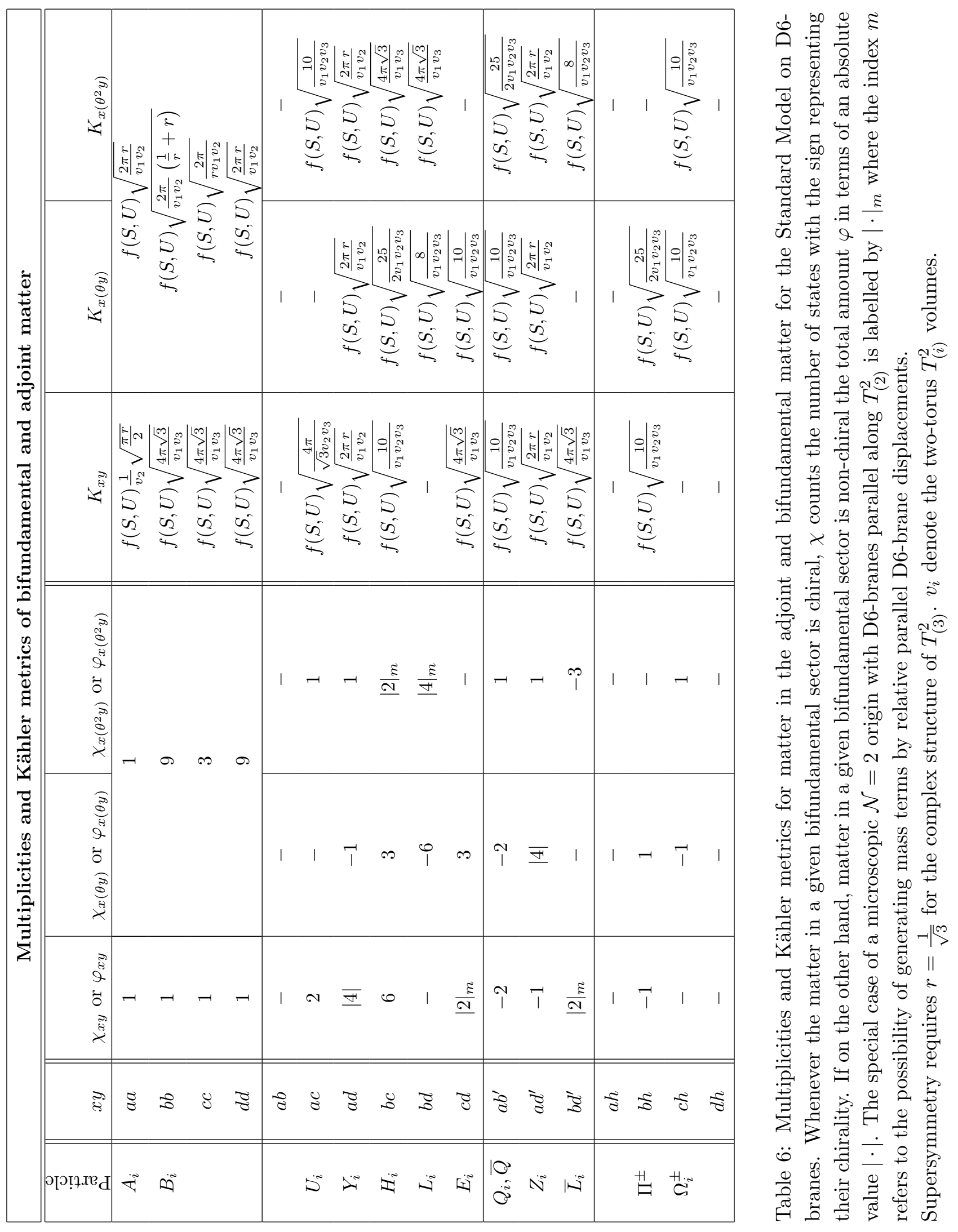




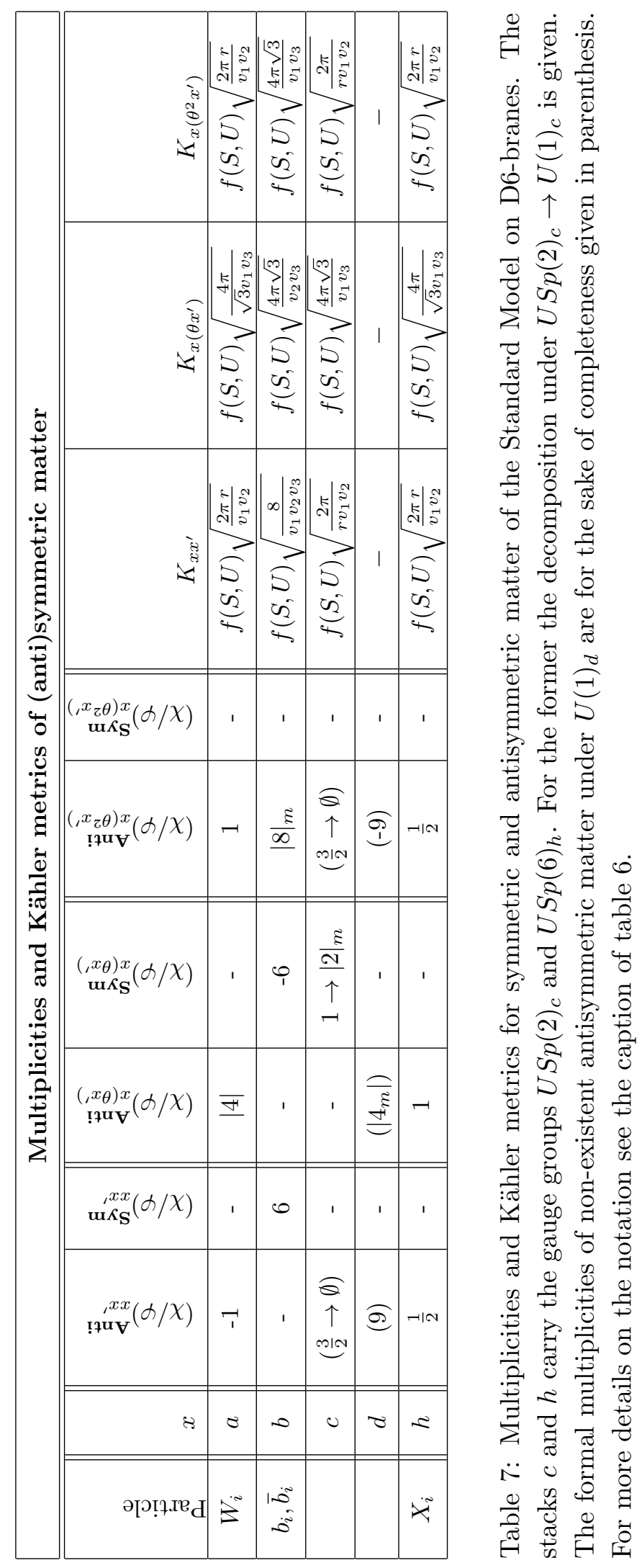




\section{B Tables with suppression factors of three-point in- teractions}

All relevant allowed three-point interactions are listed in this appendix. An allowed threepoint coupling corresponds to a cyclically ordered sequence $[x y]+[y z]+[z x] \rightsquigarrow[x, y, z]$. The oriented strings then form a closed triangle, and the corresponding interaction term is gauge invariant and produced by worldsheet instantons sweeping the area.

\begin{tabular}{|c|c|c|c|c|}
\hline \multicolumn{5}{|c|}{ Lepton Yukawa couplings before breaking $U S p(2)_{c} \rightarrow U(1)_{c}$} \\
\hline $\begin{array}{c}\text { sequence } \\
{[x, y, z]} \\
\end{array}$ & $\begin{array}{c}\text { enclosed } \\
\text { area } \\
\end{array}$ & $\begin{array}{c}\text { triangle } \\
\text { (or point) } \\
\end{array}$ & coupling & $\begin{array}{c}\text { Kähler factor } \\
\left(K_{x y} K_{y z} K_{z x}\right)^{-1 / 2}\end{array}$ \\
\hline \multirow[t]{6}{*}[c,(\thetad),b]{} & 0 & $\{4, i, 3\}$ & $E_{i} L_{i} H_{i}, i \in\{1,2,3\}$ & \multirow[t]{6}{*}{$\left(\frac{v_{1} v_{2} v_{3}}{f(S, U)^{2}}\right)^{3 / 4} \frac{1}{2(50)^{\frac{1}{4}}}$} \\
\hline & $\frac{1}{12} \mathcal{A}_{(1)}$ & $\left\{\left[\left(R, R^{\prime}\right), 4,5\right], i, 3\right\}$ & $E_{i} L_{3+i} H_{3+i}, i \in\{1,2,3\}$ & \\
\hline & $\frac{1}{6} \mathcal{A}_{(2)}$ & $\{4,[1,2,3], 3\}$ & $\begin{array}{c}E_{i} L_{j} H_{k} \text { with }(i, j, k) \\
\text { permutations of }(1,2,3)\end{array}$ & \\
\hline & $\frac{1}{12} \mathcal{A}_{(1)}+\frac{1}{6} \mathcal{A}_{(2)}$ & $\left\{\left[\left(R, R^{\prime}\right), 4,5\right],[1,2,3], 3\right\}$ & $\begin{aligned} & E_{i} L_{j} H_{k} \text { with } \\
(i, j, k)= & (1, \underline{5,6}),(2, \underline{4,6}),(3, \underline{4,5})\end{aligned}$ & \\
\hline & $\frac{1}{3} \mathcal{A}_{(1)}$ & $\left\{\left[4,\left(R, R^{\prime}\right), 4\right], i, 3\right\}$ & $E_{i} L_{3+i} H_{i}, i \in\{1,2,3\}$ & \\
\hline & $\frac{1}{3} \mathcal{A}_{(1)}+\frac{1}{6} \mathcal{A}_{(2)}$ & $\left\{\left[\left(R, R^{\prime}\right), 4,5\right],[1,2,3], 3\right\}$ & $\begin{array}{c}E_{i} L_{j} H_{k-3} \text { with } \\
(i, j, k)=(1, \underline{5,6}),(2, \underline{4,6}),(3, \underline{4,5})\end{array}$ & \\
\hline
\end{tabular}

Table 8: Three-point couplings between leptons and Higgses which are allowed by charge selection rules and the existence of triangular worldsheets. The sizes of triangular worldsheets are given before breaking the right-symmetric group $U S p(2)_{c} \rightarrow U(1)_{c}$ by a continuous displacement $\sigma_{c}^{2}$ as discussed in section 3.3.2. 


\begin{tabular}{|c|c|c|c|c|}
\hline \multicolumn{5}{|c|}{ Quark Yukawa couplings before breaking $U S p(2)_{c} \rightarrow U(1)_{c}$} \\
\hline $\begin{array}{c}\text { sequence } \\
{[x, y, z]}\end{array}$ & $\begin{array}{c}\text { enclosed } \\
\text { area }\end{array}$ & $\begin{array}{l}\text { triangle } \\
\text { (or point) }\end{array}$ & coupling & $\begin{array}{c}\text { Kähler f actor } \\
\left(K_{x y} K_{y z} K_{z x}\right)^{-1 / 2}\end{array}$ \\
\hline \multirow[t]{4}{*}[b^{\prime},a,(\theta^{2}c)]{} & 0 & $\{4,1,3\}$ & $Q_{1} U_{3} H_{1}$ & \multirow[t]{4}{*}{$\overline{\left(\frac{v_{1} v_{2} v_{3}}{f(S, U)^{2}}\right)^{3 / 4} \frac{1}{(10)^{\frac{3}{4}}}}$} \\
\hline & $\frac{1}{12} \mathcal{A}_{(2)}$ & $\left\{4,\left[\left(P, P^{\prime}\right), 1, i\right], 3\right\}$ & $Q_{2} U_{3} H_{i}, i \in\{2,3\}$ & \\
\hline & $\frac{1}{4} \mathcal{A}_{(1)}$ & $\{[4,6,4], 1,3\}$ & $Q_{1} U_{3} H_{4}$ & \\
\hline & $\frac{1}{4} \mathcal{A}_{(1)}+\frac{1}{12} \mathcal{A}_{(2)}$ & $\left\{[4,6,4],\left[\left(P, P^{\prime}\right), 1, i\right], 3\right\}$ & $Q_{2} U_{3} H_{3+i}, i \in\{2,3\}$ & \\
\hline \multirow[t]{4}{*}[(\thetab^{\prime}),a,(\theta^{2}c)]{} & 0 & $\{4,1,3\}$ & $Q_{4} U_{3} H_{7}$ & \multirow[t]{4}{*}{$\left(\frac{v_{1} v_{2} v_{3}}{f(S, U)^{2}}\right)^{3 / 4} \frac{1}{5(2)^{\frac{1}{4}}}$} \\
\hline & $\frac{1}{6} \mathcal{A}_{(2)}$ & $\{4,[1, i, 1], 3\}$ & $Q_{4} U_{3} H_{6+i}, i \in\{2,3\}$ & \\
\hline & $\frac{1}{4} \mathcal{A}_{(1)}$ & $\{[4,5,4], 1,3\}$ & $Q_{3} U_{3} H_{7}$ & \\
\hline & $\frac{1}{4} \mathcal{A}_{(1)}+\frac{1}{6} \mathcal{A}_{(2)}$ & $\{[4,5,4],[1, i, 1], 3\}$ & $Q_{3} U_{3} H_{6+i}, i \in\{2,3\}$ & \\
\hline \multirow[t]{3}{*}[(\thetab^{\prime}),a,c]{} & 0 & $\begin{array}{l}\{5,1,3\} \\
\{4,1,3\}\end{array}$ & $\begin{array}{l}Q_{3} U_{1} H_{1} \\
Q_{4} U_{1} H_{4}\end{array}$ & \multirow[t]{3}{*}{$\left(\frac{v_{1} v_{2} v_{3}}{f(S, U)^{2}}\right)^{3 / 4} \frac{(3)^{\frac{1}{8}}}{2\left(25 \pi v_{1}\right)^{\frac{1}{4}}}$} \\
\hline & \multirow[t]{2}{*}{$\frac{1}{12} \mathcal{A}_{(2)}$} & $\left\{5,\left[\left(P, P^{\prime}\right), 1, i\right], 3\right\}$ & $Q_{3} U_{2} H_{5-i}, i \in\{2,3\}$ & \\
\hline & & $\left\{4,\left[\left(P, P^{\prime}\right), 1, i\right], 3\right\}$ & $Q_{4} U_{2} H_{8-i}, i \in\{2,3\}$ & \\
\hline
\end{tabular}

Table 9: Three-point couplings between quarks and Higgses before breaking the right-symmetric group. 


\begin{tabular}{|c|c|c|c|c|}
\hline \multicolumn{5}{|c|}{ Couplings between leptons or Higgses and symmetric matter } \\
\hline $\begin{array}{c}\text { sequence } \\
{[x, y, z]}\end{array}$ & $\begin{array}{c}\text { enclosed } \\
\text { area }\end{array}$ & $\begin{array}{l}\text { triangle } \\
\text { (or point) }\end{array}$ & coupling & $\begin{array}{c}\text { Kähler factor } \\
\left(K_{x y} K_{y z} K_{z x}\right)^{-1 / 2}\end{array}$ \\
\hline \multirow[t]{8}{*}[b^{\prime},(\thetad),b]{} & 0 & $\left\{\left(R, R^{\prime}\right), i, 3\right\}$ & $\bar{L}_{i} b_{i} L_{3+i}, i \in\{1,2,3\}$ & \multirow[t]{8}{*}{$\overline{\left(\frac{v_{1} v_{2} v_{3}}{f(S, U)^{2}}\right)^{3 / 4} \frac{1}{4(2)^{\frac{1}{4}}}}$} \\
\hline & $\frac{1}{6} \mathcal{A}_{(2)}$ & $\left\{\left(R, R^{\prime}\right),[1,2,3], 3\right\}$ & $\begin{array}{c}\bar{L}_{i} b_{j} L_{k} \text { with }(i, j, k)= \\
(\underline{2,3}, 4),(\underline{1,3}, 5),(\underline{1,2}, 6)\end{array}$ & \\
\hline & $\frac{1}{4} \mathcal{A}_{(3)}$ & $\left\{\left(R, R^{\prime}\right), i,[3,1,3]\right\}$ & $\left.\overline{\bar{L}_{i} b_{3+i} L_{3+i}, i \in\{1,2}, 3\right\}$ & \\
\hline & $\frac{1}{6} \mathcal{A}_{(2)}+\frac{1}{4} \mathcal{A}_{(3)}$ & $\left\{\left(R, R^{\prime}\right),[1,2,3],[3,1,3]\right\}$ & $\begin{array}{c}\bar{L}_{i} b_{3+j} L_{k} \text { with }(i, j, k)= \\
(\underline{2,3}, 4),(\underline{1,3}, 5),(\underline{1,2}, 6)\end{array}$ & \\
\hline & $\frac{1}{6} \mathcal{A}_{(1)}$ & $\left\{\left[R, R^{\prime}, 4\right], i, 3\right\}$ & $\bar{L}_{i} b_{i} L_{i}, i \in\{1,2,3\}$ & \\
\hline & $\frac{1}{6} \mathcal{A}_{(1)}+\frac{1}{6} \mathcal{A}_{(2)}$ & $\left\{\left[R, R^{\prime}, 4\right],[1,2,3], 3\right\}$ & $\begin{array}{l}\bar{L}_{i} b_{j} L_{k} \text { with }(i, j, k)= \\
(\underline{2,3}, 1),(\underline{1,3}, 2),(\underline{1,2}, 3)\end{array}$ & \\
\hline & $\frac{1}{6} \mathcal{A}_{(1)}+\frac{1}{4} \mathcal{A}_{(3)}$ & $\left\{\left[R, R^{\prime}, 4\right], i,[3,1,3]\right\}$ & $\bar{L}_{i} b_{3+i} L_{i}, i \in\{1,2,3\}$ & \\
\hline & $\frac{1}{6} \mathcal{A}_{(1)}+\frac{1}{6} \mathcal{A}_{(2)}+\frac{1}{4} \mathcal{A}_{(3)}$ & $\left\{\left[R, R^{\prime}, 4\right],[1,2,3],[3,1,3]\right\}$ & $\begin{array}{l}\bar{L}_{i} b_{3+j} L_{k} \text { with }(i, j, k)= \\
(\underline{2,3}, 1),(\underline{1,3}, 2),(\underline{1,2}, 3)\end{array}$ & \\
\hline \multirow[t]{8}{*}[c,(\thetab^{\prime}),b]{} & \multirow[t]{2}{*}{0} & $\{4, i, 3\}$ & $\begin{array}{c}H_{j} \bar{b}_{i} H_{i} \text { with }(i, j)= \\
(1,4),(2,6),(3,5)\end{array}$ & \multirow[t]{8}{*}{$\left(\frac{v_{1} v_{2} v_{3}}{f(S, U)^{2}}\right)^{3 / 4} \frac{1}{2\left(25 \pi \sqrt{3} v_{1}\right)^{\frac{1}{4}}}$} \\
\hline & & $\{5, i, 3\}$ & $\begin{array}{c}H_{j} \bar{b}_{i} H_{3+i} \text { with }(i, j)= \\
(1,1),(2,3),(3,2)\end{array}$ & \\
\hline & \multirow[t]{2}{*}{$\frac{1}{6} \mathcal{A}_{(2)}$} & $\{4,[1,2,3], 3\}$ & $\begin{array}{l}H_{i} \bar{b}_{j} H_{k} \text { with }(i, j, k)= \\
(4, \underline{2,3}),(5, \underline{1,2}),(6, \underline{1,3})\end{array}$ & \\
\hline & & $\{5,[1,2,3], 3\}$ & $\begin{array}{l}H_{i} \bar{b}_{j} H_{k} \text { with }(i, j, k)= \\
(\underline{2,3}, 4),(\underline{1,2}, 5),(\underline{1,3}, 6)\end{array}$ & \\
\hline & \multirow[t]{2}{*}{$\frac{1}{4} \mathcal{A}_{(3)}$} & $\{4, i,[3,1,3]\}$ & $\begin{array}{c}H_{j} \bar{b}_{3+i} H_{i} \text { with }(i, j)= \\
(1,4),(2,6),(3,5)\end{array}$ & \\
\hline & & $\{5, i,[3,1,3]\}$ & $\begin{array}{c}H_{j} \bar{b}_{i} H_{i} \text { with }(i, j)= \\
(4,1),(5,3),(6,2)\end{array}$ & \\
\hline & \multirow[t]{2}{*}{$\frac{1}{6} \mathcal{A}_{(2)}+\frac{1}{4} \mathcal{A}_{(3)}$} & $\{4,[1,2,3],[3,1,3]\}$ & $\begin{array}{c}H_{i} \bar{b}_{3+j} H_{k} \text { with }(i, j, k)= \\
(4, \underline{2,3}),(5, \underline{1,2}),(6, \underline{1,3})\end{array}$ & \\
\hline & & $\{5,[1,2,3],[3,1,3]\}$ & $\begin{array}{c}H_{i} \bar{b}_{3+j} H_{k} \text { with }(i, j, k)= \\
(2,3,4),(1,2,5),(1,3,6)\end{array}$ & \\
\hline
\end{tabular}

Table 10: Three-point couplings between the symmetrics $\bar{b}_{i}$ of $U(2)_{b}$ and the leptonic $L_{j}$ and $\bar{L}_{j}$ fields as well as the symmetrics $b_{i}$ and the Higgs fields $H_{j}$. 


\begin{tabular}{|c|c|c|c|c|}
\hline \multicolumn{5}{|c|}{ Couplings between quarks and adjoint matter } \\
\hline $\begin{array}{l}\text { sequence } \\
{[x, y, z]}\end{array}$ & $\begin{array}{c}\text { enclosed } \\
\text { area }\end{array}$ & $\begin{array}{l}\text { triangle } \\
\text { (or point) }\end{array}$ & coupling & $\begin{array}{c}\text { Kähler factor } \\
\left(K_{x y} K_{y z} K_{z x}\right)^{-1 / 2}\end{array}$ \\
\hline \multirow[t]{2}{*}[(\thetaa),b^{\prime},a]{} & $\frac{1}{8} \mathcal{A}_{(1)}$ & $\{[4,5,6], 1,3\}$ & $\bar{Q} Q_{1} A_{2}$ & \multirow[t]{2}{*}{$\left(\frac{v_{1} v_{2} v_{3}}{f(S, U)^{2}}\right)^{3 / 4} \frac{1}{\left(250 \pi r v_{3}\right)^{\frac{1}{4}}}$} \\
\hline & $\frac{1}{8} \mathcal{A}_{(1)}+\frac{1}{4} \mathcal{A}_{(2)}$ & $\{[4,5,6],[1, P, 1], 3\}$ & $\bar{Q} Q_{2} A_{2}$ & \\
\hline \multirow[t]{2}{*}[(\theta^{2}a),(\thetab^{\prime}),a]{} & 0 & $\{4,1,3\}$ & $\bar{Q} Q_{4} A_{2}$ & \multirow[t]{2}{*}{$\left(\frac{v_{1} v_{2} v_{3}}{f(S, U)^{2}}\right)^{3 / 4} \frac{1}{\left(250 \pi r v_{3}\right)^{\frac{1}{4}}}$} \\
\hline & $\frac{1}{4} \mathcal{A}_{(1)}$ & $\{[4,5,4], 1,3\}$ & $\bar{Q} Q_{3} A_{2}$ & \\
\hline \multirow[t]{2}{*}[(\thetaa),b^{\prime},(\thetab^{\prime})]{} & $\begin{array}{l}\frac{1}{24} \mathcal{A}_{(1)} \\
\frac{3}{8} \mathcal{A}_{(1)} \\
\end{array}$ & $\begin{array}{c}\left\{\left[6,\left(R, R^{\prime}\right), 5\right], 1,3\right\} \\
\{[5,6,4], 1,3\} \\
\end{array}$ & $\begin{array}{c}\bar{Q} B_{k} Q_{1} \\
\text { with } k=5,8 \\
\bar{Q} B_{2} Q_{1}\end{array}$ & \multirow[t]{2}{*}{$\left(\frac{v_{1} v_{2} v_{3}}{f(S, U)^{2}}\right)^{3 / 4} \frac{1}{\left(250 \pi(r+1 / r) v_{3}\right)^{\frac{1}{4}}}$} \\
\hline & $\begin{array}{l}\frac{1}{24} \mathcal{A}_{(1)}+\frac{1}{12} \mathcal{A}_{(2)} \\
\frac{3}{8} \mathcal{A}_{(1)}+\frac{1}{12} \mathcal{A}_{(2)}\end{array}$ & $\begin{array}{c}\left\{\left[6,\left(R, R^{\prime}\right), 5\right],[1,3,4], 3\right\} \\
\left\{\left[6,\left(R, R^{\prime}\right), 5\right],[1,2,4], 3\right\} \\
\quad\{[5,6,4],[1,3,4], 3\} \\
\quad\{[5,6,4],[1,2,4], 3\} \\
\end{array}$ & $\begin{array}{c}\bar{Q} B_{k} Q_{2} \\
\text { with } k=6,9 \\
\bar{Q} B_{k} Q_{2} \\
\text { with } k=7,10 \\
\bar{Q} B_{3} Q_{2} \\
\bar{Q} B_{4} Q_{2}\end{array}$ & \\
\hline \multirow[t]{6}{*}[(\theta^{2}a),(\thetab^{\prime}),b^{\prime}]{} & 0 & $\{4,1,3\}$ & $\bar{Q} B_{2} Q_{3}$ & \multirow[t]{6}{*}{$\left(\frac{v_{1} v_{2} v_{3}}{f(S, U)^{2}}\right)^{3 / 4} \frac{1}{\left(250 \pi(r+1 / r) v_{3}\right)^{\frac{1}{4}}}$} \\
\hline & $\frac{1}{6} \mathcal{A}_{(2)}$ & $\begin{array}{l}\{4,[1,2,1], 3\} \\
\{4,[1,3,1], 3\}\end{array}$ & $\begin{array}{l}\bar{Q} B_{3} Q_{3} \\
\bar{Q} B_{4} Q_{3}\end{array}$ & \\
\hline & $\frac{1}{12} \mathcal{A}_{(1)}$ & $\left\{\left[4,\left(R, R^{\prime}\right), 6\right], 1,3\right\}$ & $\begin{array}{c}\bar{Q} B_{k} Q_{4} \\
\text { with } k=5,8\end{array}$ & \\
\hline & $\frac{1}{12} \mathcal{A}_{(1)}+\frac{1}{6} \mathcal{A}_{(2)}$ & $\begin{array}{l}\left\{\left[4,\left(R, R^{\prime}\right), 6\right],[1,2,1], 3\right\} \\
\left\{\left[4,\left(R, R^{\prime}\right), 6\right],[1,3,1], 3\right\}\end{array}$ & $\begin{array}{c}\bar{Q} B_{k} Q_{4} \\
\text { with } k=6,9 \\
\bar{Q} B_{k} Q_{4} \\
\text { with } k=7,10\end{array}$ & \\
\hline & $\frac{3}{8} \mathcal{A}_{(1)}$ & $\left\{\left[4,\left(R, R^{\prime}\right), 4\right], 1,3\right\}$ & $\begin{array}{c}\bar{Q} B_{k} Q_{3} \\
\text { with } k=5,8\end{array}$ & \\
\hline & $\frac{3}{8} \mathcal{A}_{(1)}+\frac{1}{6} \mathcal{A}_{(2)}$ & $\begin{array}{l}\left\{\left[4,\left(R, R^{\prime}\right), 4\right],[1,2,1], 3\right\} \\
\left\{\left[4,\left(R, R^{\prime}\right), 4\right],[1,3,1], 3\right\}\end{array}$ & $\begin{array}{c}\bar{Q} B_{k} Q_{3} \\
\text { with } k=6,9 \\
\bar{Q} B_{k} Q_{3} \\
\text { with } k=7,10\end{array}$ & \\
\hline
\end{tabular}

Table 11: Three-point couplings between the adjoints of $U(3)_{a}$ and $U(2)_{b}$ and the quark $Q_{i}$ and $\bar{Q}$ fields. 


\begin{tabular}{|c|c|c|c|c|}
\hline \multicolumn{5}{|c|}{ Couplings of right-handed vector-like quarks to adjoint matter } \\
\hline $\begin{array}{c}\text { sequence } \\
{[x, y, z]}\end{array}$ & $\begin{array}{c}\text { enclosed } \\
\text { area }\end{array}$ & $\begin{array}{l}\text { triangle } \\
\text { (or point) }\end{array}$ & coupling & $\begin{array}{c}\text { Kähler factor } \\
\left(K_{x y} K_{y z} K_{z x}\right)^{-1 / 2}\end{array}$ \\
\hline$\left[\left(\theta a^{\prime}\right), a,\left(\theta a^{\prime}\right)\right]$ & 0 & $\{\|=[4,5]\|,, \|=[2,3]\}$ & $\begin{array}{c}W_{i} A_{1} W_{j} \\
i=3,5 ; j=4,6\end{array}$ & $\left(\frac{v_{1} v_{2} v_{3}}{f(S, U)^{2}}\right)^{3 / 4}\left(\frac{3}{8 \pi^{3} r v_{1} v_{2} v_{3}}\right)^{1 / 4}$ \\
\hline$\left[a^{\prime}, a,(\theta a)\right]$ & $\frac{1}{8} \mathcal{A}_{(1)}$ & $\{[4,5,6], 1, \|=[23]\}$ & $W_{1} A_{2} W_{2}$ & $\left(\frac{v_{1} v_{2} v_{3}}{f(S, U)^{2}}\right)^{3 / 4} \frac{1}{\left(2 \pi r v_{3}\right)^{\frac{3}{4}}}$ \\
\hline$\left[\left(\theta a^{\prime}\right),(\theta a), a\right]$ & 0 & $\{5,1, \|=[2,3]\}$ & $\begin{array}{c}W_{1} A_{2} W_{j} \\
j=4,6\end{array}$ & $\left(\frac{v_{1} v_{2} v_{3}}{f(S, U)^{2}}\right)^{3 / 4}\left(\frac{\sqrt{3}}{16 \pi^{3} r^{2} v_{2} v_{3}^{2}}\right)^{1 / 4}$ \\
\hline$\left[\left(\theta a^{\prime}\right), a,\left(\theta^{2} a\right)\right]$ & 0 & $\{4,1, \|=[2,3]\}$ & $\begin{array}{c}W_{i} A_{2} W_{2} \\
i=3,5\end{array}$ & $\left(\frac{v_{1} v_{2} v_{3}}{f(S, U)^{2}}\right)^{3 / 4}\left(\frac{\sqrt{3}}{16 \pi^{3} r^{2} v_{2} v_{3}^{2}}\right)^{1 / 4}$ \\
\hline$\left[a,\left(\theta^{2} d\right),(\theta a)\right]$ & 0 & $\{5,1, \|=[23]\}$ & $Y_{6} Y_{5} A_{2}$ & 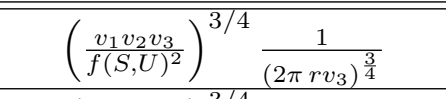 \\
\hline \multirow[t]{2}{*}[a,d,(\theta^{2}a)]{} & $\frac{1}{16} \mathcal{A}_{(1)}$ & $\left\{\left[4, U^{\prime}, 6\right], 1, \|=[23]\right\}$ & $Y_{2} Y_{5} A_{2}$ & \multirow[t]{2}{*}{$\left(\frac{v_{1} v_{2} v_{3}}{f(S, U)^{2}}\right)^{3 / 4} \frac{1}{\left(2 \pi r v_{3}\right)^{\frac{3}{4}}}$} \\
\hline & $\frac{1}{16} \mathcal{A}_{(1)}+\frac{1}{4} \mathcal{A}_{(2)}$ & $\left\{\left[4, U^{\prime}, 6\right],[1,1, P], \|=[23]\right\}$ & $Y_{4} Y_{5} A_{2}$ & \\
\hline \multirow[t]{2}{*}[d,a,(\thetaa)]{} & $\frac{1}{16} \mathcal{A}_{(1)}$ & $\{[5, U, 6], 1, \|=[23]\}$ & $Y_{1} A_{2} Y_{6}$ & \multirow[t]{2}{*}{$\left(\frac{v_{1} v_{2} v_{3}}{f(S, U)^{2}}\right)^{3 / 4} \frac{1}{\left(2 \pi r v_{3}\right)^{\frac{3}{4}}}$} \\
\hline & $\frac{1}{16} \mathcal{A}_{(1)}+\frac{1}{4} \mathcal{A}_{(2)}$ & $\{[5, U, 6],[1,1, P], \|=[23]\}$ & $Y_{3} A_{2} Y_{6}$ & \\
\hline \multirow[t]{2}{*}[d,a,a]{} & 0 & $\left\{\left(U, U^{\prime}\right), 1, \|=[23]\right\}$ & $Y_{1} A_{1} Y_{2}$ & \multirow[t]{2}{*}{$\left(\frac{v_{1} v_{2} v_{3}}{f(S, U)^{2}}\right)^{3 / 4}\left(\frac{v_{2}}{2 \pi^{3} r^{3} v_{1} v_{3}^{3}}\right)^{\frac{1}{4}}$} \\
\hline & 0 & $\left\{\left(U, U^{\prime}\right), P, \|=[23]\right\}$ & $Y_{3} A_{1} Y_{4}$ & \\
\hline$\left[d^{\prime}, a,(\theta a)\right]$ & 0 & $\{5,1, \|=[23]\}$ & $Z_{1} A_{2} Z_{6}$ & $\left(\frac{v_{1} v_{2} v_{3}}{f(S, U)^{2}}\right)^{3 / 4} \frac{1}{\left(2 \pi r v_{3}\right)^{\frac{3}{4}}}$ \\
\hline \multirow[t]{2}{*}[a,(\thetad^{\prime}),(\thetaa)]{} & $\frac{1}{16} \mathcal{A}_{(1)}$ & $\{[5, U, 6], 1, \|=[23]\}$ & $Z_{2} Z_{1} A_{2}$ & \multirow[t]{2}{*}{$\left(\frac{v_{1} v_{2} v_{3}}{f(S, U)^{2}}\right)^{3 / 4} \frac{1}{\left(2 \pi r v_{3}\right)^{\frac{3}{4}}}$} \\
\hline & $\frac{1}{16} \mathcal{A}_{(1)}+\frac{1}{4} \mathcal{A}_{(2)}$ & $\{[5, U, 6],[1,1, P], \|=[23]\}$ & $Z_{4} Z_{1} A_{2}$ & \\
\hline \multirow[t]{2}{*}[(\thetad^{\prime}),a,(\theta^{2}a)]{} & $\frac{1}{16} \mathcal{A}_{(1)}$ & $\left\{\left[4, U^{\prime}, 6\right], 1, \|=[23]\right\}$ & $Z_{3} A_{2} Z_{6}$ & \multirow[t]{2}{*}{$\left(\frac{v_{1} v_{2} v_{3}}{f(S, U)^{2}}\right)^{3 / 4} \frac{1}{\left(2 \pi r v_{3}\right)^{\frac{3}{4}}}$} \\
\hline & $\frac{1}{16} \mathcal{A}_{(1)}+\frac{1}{4} \mathcal{A}_{(2)}$ & $\left\{\left[4, U^{\prime}, 6\right],[1,1, P], \|=[23]\right\}$ & $Z_{5} A_{2} Z_{6}$ & \\
\hline \multirow[t]{2}{*}[(\thetad^{\prime}),a,a]{} & 0 & $\left\{\left(U, U^{\prime}\right), 1, \|=[23]\right\}$ & $Z_{3} A_{1} Z_{2}$ & \multirow[t]{2}{*}{$\left(\frac{v_{1} v_{2} v_{3}}{f(S, U)^{2}}\right)^{3 / 4}\left(\frac{v_{2}}{2 \pi^{3} r^{3} v_{1} v_{3}^{3}}\right)^{\frac{1}{4}}$} \\
\hline & 0 & $\left\{\left(U, U^{\prime}\right), P, \|=[23]\right\}$ & $Z_{5} A_{1} Z_{4}$ & \\
\hline
\end{tabular}

Table 12: Three-point couplings between the adjoints of $U(3)_{a}$ and various types of vector-like quarks with exotic $B-L$ charge. 


\begin{tabular}{|c|c|c|c|c|}
\hline \multicolumn{5}{|c|}{ Couplings of symmetric and adjoint matter of $U(2)_{b}$} \\
\hline $\begin{array}{c}\text { sequence } \\
{[x, y, z]}\end{array}$ & $\begin{array}{c}\text { enclosed } \\
\text { area }\end{array}$ & $\begin{array}{l}\text { triangle } \\
\text { (or point) }\end{array}$ & coupling & $\begin{array}{c}\text { Kähler factor } \\
\left(K_{x y} K_{y z} K_{z x}\right)^{-1 / 2}\end{array}$ \\
\hline \multirow[t]{3}{*}[b,b^{\prime},(\thetab^{\prime})]{} & 0 & $\begin{array}{l}\left\{\left(R, R^{\prime}\right), i, 3\right\} \\
\left\{\left(R, R^{\prime}\right), i, 1\right\}\end{array}$ & $\begin{array}{c}b_{i} B_{4+i} \bar{b}_{i}, b_{i} B_{7+i} \bar{b}_{i} \\
b_{3+i} B_{4+i} \bar{b}_{3+i}, b_{3+i} B_{7+i} \bar{b}_{3+i} \\
i=1,2,3\end{array}$ & $\left(\frac{v_{1} v_{2} v_{3}}{f(S, U)^{2}}\right)^{3 / 4} \frac{1}{\left(64 \pi^{2} \sqrt{3} v_{1} v_{3}\left(\frac{1}{r}+r\right)\right)^{\frac{1}{4}}}$ \\
\hline & \multirow[t]{2}{*}{$\frac{1}{6} \mathcal{A}_{(2)}$} & $\left\{\left(R, R^{\prime}\right),[1,2,3], 3\right\}$ & $\begin{array}{c}b_{i} B_{j} \bar{b}_{k}, b_{i} B_{3+j} \bar{b}_{k} \\
\text { with }(i j k)= \\
(163),(172),(253), \\
(271),(352),(361)\end{array}$ & \\
\hline & & $\left\{\left(R, R^{\prime}\right),[1,2,3], 1\right\}$ & $\begin{array}{c}b_{i} B_{j} \bar{b}_{k}, b_{i} B_{3+j} \bar{b}_{k} \\
\text { with }(i j k)= \\
(466),(475),(556), \\
(574),(655),(664)\end{array}$ & \\
\hline
\end{tabular}

Table 13: Three-point couplings between the symmetrics and adjoints of $U(2)_{b}$.

\begin{tabular}{|c||c|c|c|c|}
\hline \multicolumn{5}{|c|}{ Three-point couplings of hidden sector fields } \\
\hline \hline $\begin{array}{c}\text { sequence } \\
{[x, y, z]}\end{array}$ & $\begin{array}{c}\text { enclosed } \\
\text { area }\end{array}$ & $\begin{array}{c}\text { triangle } \\
\text { (or point) }\end{array}$ & coupling & $\begin{array}{c}\text { Kähler factor } \\
\left(K_{x y} K_{y z} K_{z x}\right)^{-1 / 2}\end{array}$ \\
\hline$[(\theta c), h, b]$ & $\frac{1}{16} \mathcal{A}_{(1)}+\frac{1}{8} \mathcal{A}_{(3)}$ & $\left\{\left[\left(T, T^{\prime}\right), 5,6\right], 1,[4,1,3]\right\}$ & $\Omega_{2} \Pi^{+} H_{7}$ & $\left(\frac{v_{1} v_{2} v_{3}}{f(S, U)^{2}}\right)^{3 / 4} \frac{1}{5(2)^{\frac{1}{4}}}$ \\
\cline { 2 - 4 } & $\frac{1}{8} \mathcal{A}_{(1)}+\frac{1}{6} \mathcal{A}_{(2)}+\frac{1}{4} \mathcal{A}_{(3)}$ & $\left\{\left[\left(T, T^{\prime}\right), 5,6\right],[1, i, 1],[4,1,3]\right\}$ & $\begin{array}{c}\Omega_{2} \Pi^{+} H_{6+i} \\
i \in\{2,3\}\end{array}$ & \\
\hline$[(\theta h), h, b]$ & $\frac{1}{16} \mathcal{A}_{(1)}$ & $\left\{\left[\left(T, T^{\prime}\right), 1,4\right], 1,1\right\}$ & $X_{2} \Pi^{+} \Pi^{-}$ & $\left(\frac{v_{1} v_{2} v_{3}}{f(S, U)^{2}}\right)^{3 / 4} \frac{1}{\left(250 \pi r v_{3}\right)^{\frac{1}{4}}}$ \\
\hline$\left[\left(\theta^{2} h\right),(\theta h), c\right]$ & $\frac{1}{8} \mathcal{A}_{(1)}$ & $\{[5,1,4], 1,4\}$ & $X_{2} \Omega_{1} \Omega_{2}$ & $\left(\frac{v_{1} v_{2} v_{3}}{f(S, U)^{2}}\right)^{3 / 4} \frac{1}{\left(200 \pi r v_{3}\right)^{\frac{1}{4}}}$ \\
\hline
\end{tabular}

Table 14: Three-point couplings between the hidden sector fields and Higgses as well as antisymmetrics of $U S p(6)_{h}$ and the $\Pi$ and $\Omega$ fields. 


\section{References}

[1] F. Gmeiner and G. Honecker, "Millions of Standard Models on Z-prime(6)?," JHEP, vol. 0807, p. 052, 2008.

[2] F. Gmeiner and G. Honecker, "Complete Gauge Threshold Corrections for Intersecting Fractional D6-Branes: The Z6 and Z6' Standard Models," Nucl.Phys., vol. B829, pp. 225-297, 2010. * Temporary entry*.

[3] A. M. Uranga, "Chiral four-dimensional string compactifications with intersecting Dbranes," Class.Quant.Grav., vol. 20, pp. S373-S394, 2003.

[4] R. Blumenhagen, M. Cvetič, P. Langacker, and G. Shiu, "Toward realistic intersecting D-brane models," Ann.Rev.Nucl.Part.Sci., vol. 55, pp. 71-139, 2005.

[5] R. Blumenhagen, B. Körs, D. Lüst, and S. Stieberger, "Four-dimensional String Compactifications with D-Branes, Orientifolds and Fluxes," Phys.Rept., vol. 445, pp. 1193, 2007.

[6] E. Dudas, "Orientifolds and model building," J.Phys.Conf.Ser., vol. 53, pp. 567-600, 2006.

[7] F. Marchesano, "Progress in D-brane model building," Fortsch.Phys., vol. 55, pp. 491$518,2007$.

[8] D. Lüst, "String Landscape and the Standard Model of Particle Physics," pp. 148-176, 2007.

[9] M. Cvetič and J. Halverson, "TASI Lectures: Particle Physics from Perturbative and Non-perturbative Effects in D-braneworlds," 2011.

[10] W. Buchmüller, K. Hamaguchi, O. Lebedev, and M. Ratz, "Supersymmetric standard model from the heterotic string," Phys.Rev.Lett., vol. 96, p. 121602, 2006.

[11] O. Lebedev, H. P. Nilles, S. Raby, S. Ramos-Sanchez, M. Ratz, et al., "A Minilandscape of exact MSSM spectra in heterotic orbifolds," Phys.Lett., vol. B645, pp. 8894, 2007.

[12] W. Buchmüller, K. Hamaguchi, O. Lebedev, and M. Ratz, "Supersymmetric Standard Model from the Heterotic String (II)," Nucl.Phys., vol. B785, pp. 149-209, 2007.

[13] O. Lebedev, H. P. Nilles, S. Ramos-Sanchez, M. Ratz, and P. K. Vaudrevange, "Heterotic mini-landscape. (II). Completing the search for MSSM vacua in a Z(6) orbifold," Phys.Lett., vol. B668, pp. 331-335, 2008. 
[14] T. Dijkstra, L. Huiszoon, and A. Schellekens, "Chiral supersymmetric standard model spectra from orientifolds of Gepner models," Phys.Lett., vol. B609, pp. 408-417, 2005.

[15] T. Dijkstra, L. Huiszoon, and A. Schellekens, "Supersymmetric standard model spectra from RCFT orientifolds," Nucl.Phys., vol. B710, pp. 3-57, 2005.

[16] P. Anastasopoulos, T. Dijkstra, E. Kiritsis, and A. Schellekens, "Orientifolds, hypercharge embeddings and the Standard Model," Nucl.Phys., vol. B759, pp. 83-146, 2006.

[17] V. Braun, Y.-H. He, B. A. Ovrut, and T. Pantev, "The Exact MSSM spectrum from string theory," JHEP, vol. 0605, p. 043, 2006.

[18] V. Bouchard and R. Donagi, "An SU(5) heterotic standard model," Phys.Lett., vol. B633, pp. 783-791, 2006.

[19] R. Blumenhagen, G. Honecker, and T. Weigand, "Loop-corrected compactifications of the heterotic string with line bundles," JHEP, vol. 0506, p. 020, 2005.

[20] R. Blumenhagen, G. Honecker, and T. Weigand, "Supersymmetric (non-)Abelian bundles in the Type I and SO(32) heterotic string," JHEP, vol. 0508, p. 009, 2005.

[21] R. Blumenhagen, G. Honecker, and T. Weigand, "Non-Abelian brane worlds: The Heterotic string story," JHEP, vol. 0510, p. 086, 2005.

[22] R. Blumenhagen, S. Moster, and T. Weigand, "Heterotic GUT and standard model vacua from simply connected Calabi-Yau manifolds," Nucl.Phys., vol. B751, pp. 186$221,2006$.

[23] L. B. Anderson, J. Gray, A. Lukas, and E. Palti, "Two Hundred Heterotic Standard Models on Smooth Calabi-Yau Threefolds," 2011.

[24] T. Weigand, "Lectures on F-theory compactifications and model building," Class.Quant.Grav., vol. 27, p. 214004, 2010.

[25] P. G. Camara, L. E. Ibanez, and F. Marchesano, "RR photons," JHEP, vol. 1109, p. 110, 2011.

[26] T. W. Grimm and D. V. Lopes, "The N=1 effective actions of D-branes in Type IIA and IIB orientifolds," Nucl.Phys., vol. B855, pp. 639-694, 2012.

[27] M. Kerstan and T. Weigand, "The Effective action of D6-branes in N=1 type IIA orientifolds," JHEP, vol. 1106, p. 105, 2011. 
[28] V. Braun, Y.-H. He, and B. A. Ovrut, "Yukawa couplings in heterotic standard models," JHEP, vol. 0604, p. 019, 2006.

[29] J. Derendinger, S. Ferrara, C. Kounnas, and F. Zwirner, "On loop corrections to string effective field theories: Field dependent gauge couplings and sigma model anomalies," Nucl.Phys., vol. B372, pp. 145-188, 1992. Revised version.

[30] V. Kaplunovsky and J. Louis, "On Gauge couplings in string theory," Nucl.Phys., vol. B444, pp. 191-244, 1995.

[31] D. Lüst and S. Stieberger, "Gauge threshold corrections in intersecting brane world models," Fortsch.Phys., vol. 55, pp. 427-465, 2007.

[32] N. Akerblom, R. Blumenhagen, D. Lüst, and M. Schmidt-Sommerfeld, "Thresholds for Intersecting D-branes Revisited," Phys.Lett., vol. B652, pp. 53-59, 2007.

[33] R. Blumenhagen and M. Schmidt-Sommerfeld, "Gauge Thresholds and Kaehler Metrics for Rigid Intersecting D-brane Models," JHEP, vol. 0712, p. 072, 2007.

[34] G. Honecker, "Kähler metrics and gauge kinetic functions for intersecting D6-branes on toroidal orbifolds - The complete perturbative story," Fortsch.Phys., vol. 60, pp. 243$326,2011$.

[35] G. Honecker, "Towards exact field theory results for the Standard Model on fractional D6-branes," PoS(EPS-HEP2011)129, 2011.

[36] N. Akerblom, R. Blumenhagen, D. Lüst, and M. Schmidt-Sommerfeld, "Instantons and Holomorphic Couplings in Intersecting D-brane Models," JHEP, vol. 0708, p. 044, 2007.

[37] M. Billo, M. Frau, I. Pesando, P. Di Vecchia, A. Lerda, et al., "Instanton effects in N=1 brane models and the Kähler metric of twisted matter," JHEP, vol. 0712, p. 051, 2007.

[38] M. Billo, M. Frau, I. Pesando, P. Di Vecchia, A. Lerda, et al., "Instantons in N=2 magnetized D-brane worlds," JHEP, vol. 0710, p. 091, 2007.

[39] C. Angelantonj, C. Condeescu, E. Dudas, and M. Lennek, "Stringy Instanton Effects in Models with Rigid Magnetised D-branes," Nucl.Phys., vol. B818, pp. 52-94, 2009.

[40] R. Blumenhagen, M. Cvetič, and T. Weigand, "Spacetime instanton corrections in 4D string vacua: The Seesaw mechanism for D-Brane models," Nucl.Phys., vol. B771, pp. 113-142, 2007. 
[41] L. Ibáñez and A. Uranga, "Neutrino Majorana Masses from String Theory Instanton Effects," JHEP, vol. 0703, p. 052, 2007.

[42] S. A. Abel and M. D. Goodsell, "Realistic Yukawa Couplings through Instantons in Intersecting Brane Worlds," JHEP, vol. 0710, p. 034, 2007.

[43] R. Blumenhagen, M. Cvetič, S. Kachru, and T. Weigand, "D-Brane Instantons in Type II Orientifolds," Ann.Rev.Nucl.Part.Sci., vol. 59, pp. 269-296, 2009.

[44] D. Lüst, P. Mayr, R. Richter, and S. Stieberger, "Scattering of gauge, matter, and moduli fields from intersecting branes," Nucl.Phys., vol. B696, pp. 205-250, 2004.

[45] M. Cvetič and I. Papadimitriou, "Conformal field theory couplings for intersecting D-branes on orientifolds," Phys.Rev., vol. D68, p. 046001, 2003.

[46] S. Abel and A. Owen, "Interactions in intersecting brane models," Nucl.Phys., vol. B663, pp. 197-214, 2003.

[47] D. Cremades, L. Ibáñez, and F. Marchesano, "Yukawa couplings in intersecting Dbrane models," JHEP, vol. 0307, p. 038, 2003.

[48] D. Cremades, L. Ibáñez, and F. Marchesano, "Computing Yukawa couplings from magnetized extra dimensions," JHEP, vol. 0405, p. 079, 2004.

[49] M. Berg, M. Haack, and J. U. Kang, "One-Loop Kähler Metric of D-Branes at Angles," 2011.

[50] S. A. Abel, O. Lebedev, and J. Santiago, "Flavor in intersecting brane models and bounds on the string scale," Nucl.Phys., vol. B696, pp. 141-173, 2004.

[51] N. Kitazawa, T. Kobayashi, N. Maru, and N. Okada, "Yukawa coupling structure in intersecting D-brane models," Eur.Phys.J., vol. C40, pp. 579-587, 2005.

[52] T. Higaki, N. Kitazawa, T. Kobayashi, and K.-j. Takahashi, "Flavor structure and coupling selection rule from intersecting D-branes," Phys.Rev., vol. D72, p. 086003, 2005.

[53] B. Dutta and Y. Mimura, "Lepton flavor violation in intersecting D-brane models," Phys.Lett., vol. B638, pp. 239-245, 2006.

[54] F. Gmeiner and G. Honecker, "Mapping an Island in the Landscape," JHEP, vol. 0709, p. $128,2007$.

[55] D. Bailin and A. Love, "Towards the supersymmetric standard model from intersecting D6-branes on the Z-prime(6) orientifold," Nucl.Phys., vol. B755, pp. 79-111, 2006. 
[56] D. Bailin and A. Love, "Almost the supersymmetric standard model from intersecting D6-branes on the Z(6)-prime orientifold," Phys.Lett., vol. B651, pp. 324-328, 2007.

[57] D. Bailin and A. Love, "Constructing the supersymmetric Standard Model from intersecting D6-branes on the Z(6)-prime orientifold," Nucl.Phys., vol. B809, pp. 64-109, 2009.

[58] S. Förste and G. Honecker, "Rigid D6-branes on $T^{6} /\left(Z_{2} x Z_{2 M} x \Omega R\right)$ with discrete torsion," JHEP, vol. 1101, p. 091, 2011.

[59] L. E. Ibáñez, F. Marchesano, and R. Rabadan, "Getting just the standard model at intersecting branes," JHEP, vol. 0111, p. 002, 2001.

[60] R. Blumenhagen, L. Görlich, and T. Ott, "Supersymmetric intersecting branes on the type 2A T6 / Z(4) orientifold," JHEP, vol. 0301, p. 021, 2003.

[61] G. Honecker and T. Ott, "Getting just the supersymmetric standard model at intersecting branes on the Z(6) orientifold," Phys.Rev., vol. D70, p. 126010, 2004.

[62] G. Honecker, "Chiral N=1 4-D orientifolds with D-branes at angles," Mod.Phys.Lett., vol. A19, pp. 1863-1879, 2004.

[63] F. Gmeiner, D. Lüst, and M. Stein, "Statistics of intersecting D-brane models on T**6 / Z(6)," JHEP, vol. 0705, p. 018, 2007.

[64] G. Honecker, "Chiral supersymmetric models on an orientifold of Z(4) x Z(2) with intersecting D6-branes," Nucl.Phys., vol. B666, pp. 175-196, 2003.

[65] M. Cvetič and P. Langacker, "New Grand Unified Models with Intersecting D6-branes, Neutrino Masses, and Flipped SU(5)," Nucl.Phys., vol. B776, pp. 118-137, 2007.

[66] S. Förste, G. Honecker, and R. Schreyer, "Supersymmetric Z(N) x Z(M) orientifolds in 4-D with D branes at angles," Nucl.Phys., vol. B593, pp. 127-154, 2001.

[67] G. Honecker and J. Vanhoof, "Towards the field theory of the Standard Model on fractional D6-branes on T6/Z6': Yukawa couplings and masses," Fortsch.Phys., 2012.

[68] S. Abel and A. Owen, "N point amplitudes in intersecting brane models," Nucl.Phys., vol. B682, pp. 183-216, 2004.

[69] E. Cremmer, S. Ferrara, L. Girardello, and A. Van Proeyen, "Yang-Mills Theories with Local Supersymmetry: Lagrangian, Transformation Laws and SuperHiggs Effect," Nucl.Phys., vol. B212, p. 413, 1983. 
[70] L. J. Dixon, V. Kaplunovsky, and J. Louis, "On Effective Field Theories Describing $(2,2)$ Vacua of the Heterotic String," Nucl.Phys., vol. B329, pp. 27-82, 1990.

[71] R. Blumenhagen, D. Lüst, and S. Stieberger, "Gauge unification in supersymmetric intersecting brane worlds," JHEP, vol. 07, p. 036, 2003.

[72] K. Benakli and M. Goodsell, "Two-Point Functions of Chiral Fields at One Loop in Type II," Nucl.Phys., vol. B805, pp. 72-103, 2008.

[73] M. Cvetic and T. Weigand, "A String theoretic model of gauge mediated supersymmetry beaking," 2008.

[74] M. Blaszczyk, S. Nibbelink Groot, M. Ratz, F. Ruehle, M. Trapletti, et al., "A Z2xZ2 standard model," Phys.Lett., vol. B683, pp. 340-348, 2010.

[75] S. A. Abel and M. D. Goodsell, "Intersecting brane worlds at one loop," JHEP, vol. 0602, p. 049, 2006.

[76] M. Berg, M. Haack, and B. Körs, "String loop corrections to Kähler potentials in orientifolds," JHEP, vol. 0511, p. 030, 2005.

[77] P. Anastasopoulos, I. Antoniadis, K. Benakli, M. Goodsell, and A. Vichi, "One-loop adjoint masses for non-supersymmetric intersecting branes," JHEP, vol. 1108, p. 120, 2011.

[78] P. Anastasopoulos, M. Bianchi, and R. Richter, "On closed-string twist-field correlators and their open-string descendants," 2011.

[79] J. Vanhoof, "From D6-branes to the Standard Model A field theoretic approach," Master thesis, K.U.Leuven, http://bib.kuleuven.be/cba/cba-english/searching/collection/dissertations, 2011. 